Mathematical Modelling AND ANALysis

Volume 17 Number 3, June 2012, 423-446

http://dx.doi.org/10.3846/13926292.2012.686068

(c) Vilnius Gediminas Technical University, 2012
Publisher: Taylor\&Francis and VGTU

http://www.tandfonline.com/TMMA

Print ISSN: 1392-6292

Online ISSN: 1648-3510

\title{
Solvability of Boundary Value Problems for Singular Quasi-Laplacian Differential Equations on the Whole Line*
}

\section{Yuji Liu}

\author{
Guangdong University of Business Studies \\ Guangdong Province, China \\ E-mail: liuyuji888@sohu.com
}

Received July 6, 2011; revised March 26, 2012; published online June 1, 2012

\begin{abstract}
This paper is concerned with some integral type boundary value problems associated to second order singular differential equations with quasi-Laplacian on the whole line. The emphasis is put on the one-dimensional $p$-Laplacian term $\left[\Phi\left(\rho(t) a\left(t, x(t), x^{\prime}(t)\right) x^{\prime}(t)\right)\right]^{\prime}$ involving a nonnegative function $\rho$ that may be singular at $t=0$ and such that $\int_{-\infty}^{0} \frac{d s}{\rho(s)}=\int_{0}^{+\infty} \frac{d s}{\rho(s)}=+\infty$. A Banach space and a nonlinear completely continuous operator are defined in this paper. By using the Schauder's fixed point theorem, sufficient conditions to guarantee the existence of at least one solution are established. An example is presented to illustrate the main theorem.
\end{abstract}

Keywords: second order singular differential equation with quasi-Laplacian on the whole line, integral type boundary value problem, fixed point theorem.

AMS Subject Classification: 34B10; 34B15; 35B10.

\section{Introduction}

The multi-point boundary-value problems for linear second order ordinary differential equations (ODEs) were initiated by Il'in and Moiseev [15]. Since then, more general nonlinear multi-point boundary-value problems (BVPs) were studied by several authors, see the paper $[8,9,10,19]$, the text books $[1,13,14]$, the survey papers $[11,18]$ and the references therein. However, the study of the existence of solutions of differential equations on the whole real line with nonlinear differential operators does not seem to be sufficiently developed [5].

Differential equations governed by nonlinear differential operators have been widely studied. In this setting the most investigated operator is the classical $p$ Laplacian, that is $\Phi_{p}(x)=|x|^{p-2} x$ with $p>1$, which, in recent years, has been

* Supported by the Natural Science Foundation of Guangdong province (No. S2011010001900) and the Guangdong Higher Education Foundation for High-level talents. 
generalized to other types of differential operators, that preserve the monotonicity of the $p$-Laplacian, but are not homogeneous. These more general operators, which are usually referred to as $\Phi$-Laplacian (or quasi-Laplacian), are involved in some models, e.g. in non-Newtonian fluid theory, diffusion of flows in porous media, nonlinear elasticity and theory of capillary surfaces. The related nonlinear differential equation has the form

$$
\left[\Phi\left(x^{\prime}\right)\right]^{\prime}=f\left(t, x, x^{\prime}\right), \quad t \in(-\infty,+\infty),
$$

where $\Phi: R \rightarrow R$ is an increasing homeomorphism such that $\Phi(0)=0$. More recently, equations involving other types of differential operators have been studied from a different point of view arising from other types of models, e.g. reaction diffusion equations with non-constant diffusivity and porous media equations. This leads to consider nonlinear differential operators of the type $\left[a\left(t, x, x^{\prime}\right) \Phi\left(x^{\prime}\right)\right]^{\prime}$, where $a$ is a positive continuous function. For a comprehensive bibliography on this subject, see e.g. [11, 16, 18].

In [17], the authors study a class of BVPs for the second order nonlinear ordinary differential equations on the whole line. Two theorems have been proved. The first one is established by the use of the Schauder theorem and concerns the existence of solutions, while the second one deals with the existence and uniqueness of solutions and is derived by the Banach contraction principle.

In [12], the authors study the boundary value problem $\left[a(x(t)) \Phi\left(x^{\prime}(t)\right)\right]^{\prime}=$ $f\left(t, x(t), x^{\prime}(t)\right), t \in(-\infty,+\infty), x(-\infty)=\nu_{1}, x(+\infty)=\nu_{2}$, establishing the existence and non-existence of heteroclinic solutions.

In [5], Bianconi and Papalini investigate the existence of solutions of the following boundary value problem

$$
\begin{aligned}
& {\left[\Phi\left(x^{\prime}(t)\right)\right]^{\prime}+a(t, x(t)) b\left(x(t), x^{\prime}(t)\right)=0, \quad t \in R,} \\
& \lim _{t \rightarrow-\infty} x(t)=: x(-\infty)=0, \quad \lim _{t \rightarrow+\infty} x(t)=: x(+\infty)=1,
\end{aligned}
$$

where $\Phi$ is a monotone function which generalizes the one-dimensional $p$-Laplacian operator. A criterion for the existence and non-existence of solutions of BVP (1.1) is established. In [2, 4], Avramescu and Vladimirescu study the following boundary value problem

$$
\begin{aligned}
& x^{\prime \prime}(t)+2 f(t) x^{\prime}(t)+x(t)+g(t, x(t))=0, \quad t \in R, \\
& \lim _{t \rightarrow \pm \infty} x(t)=: x( \pm \infty)=0, \quad \lim _{t \rightarrow \pm \infty} x^{\prime}(t)=: x^{\prime}( \pm \infty)=0,
\end{aligned}
$$

where $f$ and $g$ are given functions. The existence of solutions of BVP (1.2) is obtained. In [3], Avramescu and Vladimirescu study the following boundary value problem

$$
\begin{aligned}
& x^{\prime \prime}(t)+f\left(t, x(t), x^{\prime}(t)\right)=0, \quad t \in R, \\
& \lim _{t \rightarrow-\infty} x(t)=\lim _{t \rightarrow+\infty} x(t), \quad \lim _{t \rightarrow-\infty} x^{\prime}(t)=\lim _{t \rightarrow+\infty} x^{\prime}(t),
\end{aligned}
$$

under some adequate hypothesises and using the Bohnenblust-Karlin fixed point theorem, the existence of solutions of BVP (1.3) is established. 
Cabada and Cid [6] prove the solvability of the boundary value problem on the whole line

$$
\begin{aligned}
& {\left[\Phi\left(x^{\prime}(t)\right)\right]^{\prime}+f\left(t, x(t), x^{\prime}(t)\right)=0, \quad t \in R,} \\
& \lim _{t \rightarrow-\infty} x(t)=-1, \quad \lim _{t \rightarrow+\infty} x(t)=1,
\end{aligned}
$$

where $f$ is a continuous function, $\Phi:(-a, a) \rightarrow R$ is a homeomorphism with $a \in(0,+\infty)$, i.e., $\Phi$ is singular. Calamai [7] and Marcelli, Papalini [17] discuss the solvability of the following strongly nonlinear BVP:

$$
\begin{aligned}
& {\left[a(x(t)) \Phi\left(x^{\prime}(t)\right)\right]^{\prime}+f\left(t, x(t), x^{\prime}(t)\right)=0, \quad t \in R,} \\
& \lim _{t \rightarrow-\infty} x(t)=\alpha, \quad \lim _{t \rightarrow+\infty} x(t)=\beta,
\end{aligned}
$$

where $\alpha<\beta, \Phi$ is a general increasing homeomorphism with bounded domain (singular $\Phi$-Laplacian), $a$ is a positive continuous function and $f$ is a Caratheodory nonlinear function. Conditions for the existence and nonexistence of heteroclinic solutions in terms of the behavior of $y \rightarrow f(t, x, y)$ and $y \rightarrow \Phi(y)$ as $y \rightarrow 0$, and of $t \rightarrow f(t, x, y)$ as $|t| \rightarrow+\infty$ are established. The approach is based on fixed point techniques suitably combined to the method of upper and lower solutions.

Motivated by the mentioned papers, we consider the more general BVP for a second order singular differential equation on the whole line with quasiLaplacian operator

$$
\begin{aligned}
& {\left[\Phi\left(\rho(t) a\left(t, x(t), x^{\prime}(t)\right) x^{\prime}(t)\right)\right]^{\prime}+f\left(t, x(t), x^{\prime}(t)\right)=0, \quad t \in R,} \\
& \lim _{t \rightarrow-\infty} \rho(t) a\left(t, x(t), x^{\prime}(t)\right) x^{\prime}(t)-\int_{-\infty}^{+\infty} \alpha(s) x(s) d s=\int_{-\infty}^{+\infty} g\left(s, x(s), x^{\prime}(s)\right) d s \\
& \lim _{t \rightarrow+\infty} \rho(t) a\left(t, x(t), x^{\prime}(t)\right) x^{\prime}(t)+\int_{-\infty}^{+\infty} \beta(s) x^{\prime}(s) d s=\int_{-\infty}^{+\infty} h\left(s, x(s), x^{\prime}(s)\right) d s
\end{aligned}
$$

where

- $\rho \in C^{0}(R,[0,+\infty))$ with $\rho(t)>0$ for all $t \neq 0$ satisfies

$$
\int_{-\infty}^{0} d s / \rho(s)=+\infty, \quad \int_{0}^{+\infty} d s / \rho(s)=+\infty .
$$

Denote $\tau(t)=\left|\int_{0}^{t} d s / \rho(s)\right|$.

- $a: R \times R \times R \rightarrow(0,+\infty)$ is continuous and satisfies that there exist constants $m>0, M>0$ such that

$$
m \leq a(t,(1+\tau(t)) x, y / \rho(t)) \leq M, \quad t \in R, x \in R, y \in R
$$

and for each $r>0,|x|,|y| \leq r$ imply that $a(t,(1+\tau(t)) x, y / \rho(t)) \rightarrow a_{ \pm \infty}$ uniformly as $t \rightarrow \pm \infty$.

- $f, g, h$ defined on $R^{3}$ are nonnegative Caratheodory functions. 
- $\alpha, \beta: R \rightarrow[0,+\infty)$ are continuous functions satisfying

$$
\begin{aligned}
& \int_{-\infty}^{+\infty} \alpha(s) d s>0, \quad \int_{-\infty}^{+\infty} \frac{\beta(s)}{\rho(s)} d s<+\infty \\
& \int_{0}^{+\infty} \alpha(s) \int_{0}^{s} \frac{d r}{\rho(r)} d s<+\infty, \quad \int_{-\infty}^{0} \alpha(s) \int_{s}^{0} \frac{d r}{\rho(r)} d s<+\infty
\end{aligned}
$$

- $\Phi \in C^{1}(R)$ (a quasi-Laplacian operator) is continuous and strictly increasing on $R, \Phi(0)=0$ and its inverse function denoted by $\Phi^{-1}$ is continuous too, moreover $\Phi^{-1}$ satisfies that there exist constants $L>0$ and $L_{n}>0$ such that $\Phi^{-1}\left(x_{1} x_{2}\right) \leq L \Phi^{-1}\left(x_{1}\right) \Phi^{-1}\left(x_{2}\right)$ and

$$
\begin{gathered}
\Phi^{-1}\left(x_{1}+\cdots+x_{n}\right) \leq L_{n}\left[\Phi^{-1}\left(x_{1}\right)+\cdots+\Phi^{-1}\left(x_{n}\right)\right], \\
x_{i} \geq 0(i=1,2, \ldots, n) .
\end{gathered}
$$

It is well known that $\Phi(s)=|s|^{p-2} s$ with $p>1$ is called $p$-Laplacian. One sees that quasi-Laplacian contains $p$-Laplacian as special case. But $\Phi(s)=\frac{s^{3}}{1+s^{2}}$ is a quasi-Laplacian not a $p$-Laplacian.

By a solution of BVP (1.7) we mean a function $x \in C^{1}(R)$ such that

$$
\Phi\left(\rho a x^{\prime}\right): t \rightarrow \Phi\left(\rho(t) a\left(t, x(t), x^{\prime}(t)\right) x^{\prime}(t)\right)
$$

belongs to $W^{1,1}(R)$ and all equations in (1.7) are satisfied.

The purpose is to establish sufficient conditions for the existence of at least one solution of BVP (1.7). The results in this paper generalize and improve some known ones since the quasi-Laplacian term $\left[\Phi\left(\rho(t) a\left(t, x(t), x^{\prime}(t)\right) x^{\prime}(t)\right)\right]^{\prime}$ involves the nonnegative function $\rho$ that may satisfy $\rho(0)=0$.

The remainder of this paper is organized as follows: the preliminary results are given in Section 2, the main results are presented in Section 3. An example is presented in Section 4 to illustrate the prototype of the main theorem.

\section{Preliminary Results}

In this section, we present some background definitions in Banach spaces and state an important fixed point theorem. The preliminary results are given too.

Let $X$ be a Banach space. An operator $T ; X \rightarrow X$ is completely continuous if it is continuous and maps bounded sets into relatively compact sets.

Lemma 1 [Schauder]. Let $X$ be a Banach space and $\Omega \subset X$ a nonempty, bounded, open and convex subset of $X$. Let $T: \bar{\Omega} \rightarrow X$ be a completely continuous operator with $T(\partial \Omega) \subset \bar{\Omega}$. Then $T$ has a fixed point in $\Omega$.

Definition 1. $f: R \times R \times R \rightarrow R$ is called a Carathédory function if it satisfies

(i) $t \rightarrow f(t,(1+\tau(t)) x, y / \rho(t))$ is measurable for any $x, y \in R$,

(ii) $(x, y) \rightarrow f(t,(1+\tau(t)) x, y / \rho(t))$ is continuous for a.e. $t \in R$, 
(iii) for each $r>0$, there exists nonnegative function $\phi_{r} \in L^{1}(R)$ such that $|u|,|v| \leq r$ implies

$$
|f(t,(1+\tau(t)) u, v / \rho(t))| \leq \phi_{r}(t), \quad \text { a.e. } t \in R .
$$

Define

$$
X=\left\{\begin{array}{c}
x \in C^{0}(R), \rho x^{\prime} \in C^{0}(R) \\
x: R \rightarrow R: \quad t \rightarrow \frac{x(t)}{1+\tau(t)} \text { is bounded on } R \\
t \rightarrow \rho(t) x^{\prime}(t) \text { is bounded on } R
\end{array}\right\} .
$$

For $x \in X$, define the norm of $x$ by

$$
\|x\|=\max \left\{\sup _{t \in R} \frac{|x(t)|}{1+\tau(t)}, \sup _{t \in R} \rho(t)\left|x^{\prime}(t)\right|\right\} .
$$

One can prove that $X$ is a Banach space with the norm $\|x\|$ for $x \in X$.

Lemma 2. Suppose that $x \in X$. Denote

$$
\begin{aligned}
\sigma_{1} & =-\int_{-\infty}^{+\infty}\left|f\left(r, x(r), x^{\prime}(r)\right)\right| d r+\Phi\left(\frac{\int_{-\infty}^{+\infty} h\left(r, x(r), x^{\prime}(r)\right) d r}{1+\int_{-\infty}^{+\infty} \frac{\beta(r)}{\rho(r) a\left(r, x(r), x^{\prime}(r)\right)} d r}\right), \\
\sigma_{2} & =\int_{-\infty}^{+\infty}\left|f\left(r, x(r), x^{\prime}(r)\right)\right| d r+\Phi\left(\frac{\int_{-\infty}^{+\infty} h\left(r, x(r), x^{\prime}(r)\right) d r}{1+\int_{-\infty}^{+\infty} \frac{\beta(r)}{\rho(r) a\left(r, x(r), x^{\prime}(r)\right)} d r}\right) .
\end{aligned}
$$

Then there exists a unique constant $A_{x} \in\left[\sigma_{1}, \sigma_{2}\right]$ such that

$$
\begin{gathered}
\Phi^{-1}\left(A_{x}\right)+\int_{-\infty}^{+\infty} \frac{\beta(s) \Phi^{-1}\left(A_{x}+\int_{s}^{+\infty} f\left(r, x(r), x^{\prime}(r)\right) d r\right)}{\rho(s) a\left(s, x(s), x^{\prime}(s)\right)} d s \\
-\int_{-\infty}^{+\infty} h\left(r, x(r), x^{\prime}(r)\right) d r=0 .
\end{gathered}
$$

Furthermore, it holds that

$$
\left|A_{x}\right| \leq \int_{-\infty}^{+\infty}\left|f\left(r, x(r), x^{\prime}(r)\right)\right| d r+\Phi\left(\frac{\int_{-\infty}^{+\infty}\left|h\left(r, x(r), x^{\prime}(r)\right)\right| d r}{1+\int_{-\infty}^{+\infty} \frac{\beta(r)}{M \rho(r)} d r}\right)
$$

where $M$ is defined in Section 1.

Proof. Since $x \in X, f, h$ are Caratheodory functions, then

$$
\|x\|=\max \left\{\sup _{t \in R} \frac{|x(t)|}{1+\tau(t)}, \sup _{t \in R} \rho(t)\left|x^{\prime}(t)\right|\right\}=r<+\infty,
$$

and both

$$
\int_{-\infty}^{+\infty} f\left(r, x(r), x^{\prime}(r)\right) d r \quad \text { and } \quad \int_{-\infty}^{+\infty} h\left(r, x(r), x^{\prime}(r)\right) d r
$$


converge. Let

$$
\begin{aligned}
G(c)=\Phi^{-1}(c)+\int_{-\infty}^{+\infty} \frac{\beta(s) \Phi^{-1}\left(c+\int_{s}^{+\infty} f\left(r, x(r), x^{\prime}(r)\right) d r\right)}{\rho(s) a\left(s, x(s), x^{\prime}(s)\right)} d s \\
\quad-\int_{-\infty}^{+\infty} h\left(r, x(r), x^{\prime}(r)\right) d r .
\end{aligned}
$$

Since $\int_{-\infty}^{+\infty} \frac{\beta(s)}{\rho(s)} d s<+\infty$, then $G(c)$ is well defined on $R$. It is easy to see that $G(c)$ is strictly increasing on $R$. We find that

$$
\begin{aligned}
G\left(\sigma_{1}\right)= & \Phi^{-1}\left(-\int_{-\infty}^{+\infty}\left|f\left(r, x(r), x^{\prime}(r)\right)\right| d r+\Phi\left(\frac{\int_{-\infty}^{+\infty} h\left(r, x(r), x^{\prime}(r)\right) d r}{1+\int_{-\infty}^{+\infty} \frac{\beta(r)}{\rho(r) a\left(r, x(r), x^{\prime}(r)\right)} d r}\right)\right) \\
& +\int_{-\infty}^{+\infty} \frac{\beta(s)}{\rho(s) a\left(s, x(s), x^{\prime}(s)\right)} \Phi^{-1}\left(-\int_{-\infty}^{+\infty}\left|f\left(r, x(r), x^{\prime}(r)\right)\right| d r\right. \\
& \left.+\Phi\left(\frac{\int_{-\infty}^{+\infty} h\left(r, x(r), x^{\prime}(r)\right) d r}{1+\int_{-\infty}^{+\infty} \frac{\beta(r)}{\rho(r) a\left(r, x(r), x^{\prime}(r)\right)} d r}\right)+\int_{s}^{+\infty} f\left(r, x(r), x^{\prime}(r)\right) d r\right) d s \\
& -\int_{-\infty}^{+\infty} h\left(r, x(r), x^{\prime}(r)\right) d r \leq \Phi^{-1}\left(\Phi\left(\frac{\int_{-\infty}^{+\infty} h\left(r, x(r), x^{\prime}(r)\right) d r}{1+\int_{-\infty}^{+\infty} \frac{\beta(r)}{\rho(r) a\left(r, x(r), x^{\prime}(r)\right)} d r}\right)\right) \\
& +\int_{-\infty}^{+\infty} \frac{\beta(s)}{\rho(s) a\left(s, x(s), x^{\prime}(s)\right)} d s \Phi^{-1}\left(\Phi\left(\frac{\int_{-\infty}^{+\infty} h\left(r, x(r), x^{\prime}(r)\right) d r}{1+\int_{-\infty}^{+\infty} \frac{\beta(r)}{\rho(r) a\left(r, x(r), x^{\prime}(r)\right)} d r}\right)\right) \\
& -\int_{-\infty}^{+\infty} h\left(r, x(r), x^{\prime}(r)\right) d r \\
= & \frac{\int_{-\infty}^{+\infty} h\left(r, x(r), x^{\prime}(r)\right) d r}{1+\int_{-\infty}^{+\infty} \frac{\beta(r)}{\rho(r) a\left(r, x(r), x^{\prime}(r)\right)} d r}-\int_{-\infty}^{+\infty} h\left(r, x(r), x^{\prime}(r)\right) d r \\
& +\int_{-\infty}^{+\infty} \frac{\beta(s)}{\rho(s) a\left(s, x(s), x^{\prime}(s)\right)} d s \frac{\int_{-\infty}^{+\infty} h\left(r, x(r), x^{\prime}(r)\right) d r}{1+\int_{-\infty}^{+\infty} \frac{\beta(r)}{\rho(r) a\left(r, x(r), x^{\prime}(r)\right)} d r}=0 .
\end{aligned}
$$

Similarly we find that $G\left(\sigma_{2}\right) \geq 0$.

Hence there exists a unique constant $A_{x} \in\left[\sigma_{1}, \sigma_{2}\right]$ such that (2.1) holds. It is easy to see from $A_{x} \in\left[\sigma_{1}, \sigma_{2}\right]$ that $(2.2)$ holds. The proof is complete.

Define the operator $T: X \rightarrow X$ by

$$
(T x)(t)= \begin{cases}B_{x}+\int_{0}^{t} \frac{\Phi^{-1}\left(A_{x}+\int_{s}^{+\infty} f\left(r, x(r), x^{\prime}(r)\right) d r\right)}{\rho(s) a\left(s, x(s), x^{\prime}(s)\right)} d s, & t \geq 0, \\ B_{x}-\int_{t}^{0} \frac{\Phi^{-1}\left(A_{x}+\int_{s}^{+\infty} f\left(r, x(r), x^{\prime}(r)\right) d r\right)}{\rho(s) a\left(s, x(s), x^{\prime}(s)\right)} d s, & t \leq 0,\end{cases}
$$

where $A_{x}$ satisfies (2.1) and

$$
B_{x}=\frac{\Phi^{-1}\left(A_{x}+\int_{-\infty}^{+\infty} f\left(r, x(r), x^{\prime}(r)\right) d r\right)-\int_{-\infty}^{+\infty} g\left(r, x(r), x^{\prime}(r)\right) d r}{\int_{-\infty}^{+\infty} \alpha(s) d s}
$$




$$
\begin{aligned}
& -\frac{\int_{0}^{+\infty} \alpha(s) \int_{0}^{s} \frac{\Phi^{-1}\left(A_{x}+\int_{u}^{+\infty} f\left(r, x(r), x^{\prime}(r)\right) d r\right)}{\rho(u) a\left(u, x(u), x^{\prime}(u)\right)} d u d s}{\int_{-\infty}^{+\infty} \alpha(s) d s} \\
& +\frac{\int_{-\infty}^{0} \alpha(s) \int_{s}^{0} \frac{\Phi^{-1}\left(A_{x}+\int_{u}^{+\infty} f\left(r, x(r), x^{\prime}(r)\right) d r\right)}{\rho(u) a\left(u, x(u), x^{\prime}(u)\right)} d u d s}{\int_{-\infty}^{+\infty} \alpha(s) d s} .
\end{aligned}
$$

Lemma 3. The following properties hold:

(i) Tx satisfies

$$
\left\{\begin{array}{l}
{\left[\Phi\left(\rho(t) a\left(t, x(t), x^{\prime}(t)\right)(T x)^{\prime}(t)\right)\right]^{\prime}+f\left(t, x(t), x^{\prime}(t)\right)=0, \quad t \in R,} \\
\lim _{t \rightarrow-\infty} \rho(t) a\left(t, x(t), x^{\prime}(t)\right)(T x)^{\prime}(t)-\int_{-\infty}^{+\infty} \alpha(s)(T x)(s) d s \\
\quad=\int_{-\infty}^{+\infty} g\left(s, x(s), x^{\prime}(s)\right) d s, \\
\lim _{t \rightarrow+\infty} \rho(t) a\left(t, x(t), x^{\prime}(t)\right)(T x)^{\prime}(t)+\int_{-\infty}^{+\infty} \beta(s)(T x)^{\prime}(s) d s \\
\quad=\int_{-\infty}^{+\infty} h\left(s, x(s), x^{\prime}(s)\right) d s .
\end{array}\right.
$$

(ii) $T: X \rightarrow X$ is well defined.

(iii) $x \in X$ is a solution of $B V P(1.7)$ if and only if $x$ is a fixed point of $T$ in $X$.

(iv) $T$ is completely continuous.

Proof. (i) Let $x \in X$, by Lemma 2, each $A_{x}$ is uniquely determined. Hence $B_{x}$ is well defined. So $T x$ is well defined. Since $f, g, h$ are Caratheodory functions, then

$$
\|x\|=\max \left\{\sup _{t \in R} \frac{|x(t)|}{1+\tau(t)}, \sup _{t \in R} \rho(t)\left|x^{\prime}(t)\right|\right\}=r<+\infty
$$

and

$$
\int_{-\infty}^{+\infty} f\left(r, x(r), x^{\prime}(r)\right) d r, \quad \int_{-\infty}^{+\infty} g\left(r, x(r), x^{\prime}(r)\right) d r, \quad \int_{-\infty}^{+\infty} h\left(r, x(r), x^{\prime}(r)\right) d r
$$

converge. From the definitions of $A_{x}$ and $B_{x}$, we get

$$
\rho(t)(T x)^{\prime}(t)=\frac{1}{a\left(t, x(t), x^{\prime}(t)\right)} \Phi^{-1}\left(A_{x}+\int_{t}^{+\infty} f\left(r, x(r), x^{\prime}(r)\right) d r\right) .
$$

It is easy to see that $(2.5)$ holds.

(ii) From the assumptions imposed on $\alpha, \beta, \rho$, we know that $t \rightarrow(T x)(t)$ is continuous on $R$ and $(T x)(t) /(1+\tau(t))$ is bounded on $R$. Furthermore,

$$
\rho(t)(T x)^{\prime}(t)=\frac{\Phi^{-1}\left(A_{x}+\int_{t}^{+\infty} f\left(r, x(r), x^{\prime}(r)\right) d r\right)}{a\left(t, x(t), x^{\prime}(t)\right)} .
$$

It is easy to see that $t \rightarrow \rho(t)(T x)^{\prime}(t)$ is continuous on $R$ and $\rho(t)(T x)^{\prime}(t)$ is bounded on $R$. It follows that $T x \in X$. Hence $T: X \rightarrow X$ is well defined. 
(iii) It is easy to see that $x \in X$ is a solution of BVP (1.7) if and only if $x$ is a fixed point of $T$ in $X$.

(iv) The following five steps are needed (Steps 1-2 imply that $T: X \rightarrow X$ is continuous and Steps 3-5 imply that $T$ maps bounded sets into relatively compact sets). It follows that $T: X \rightarrow X$ is completely continuous.

Step 1. We prove that the function $A_{x}: X \rightarrow R$ is continuous in $x$.

Let $\left\{x_{n}\right\} \in X$ with $x_{n} \rightarrow x_{0}$ as $n \rightarrow \infty$. Let $\left\{A_{x_{n}}\right\}(n=0,1,2, \ldots)$ be constants decided by equation

$$
\begin{gathered}
\Phi^{-1}\left(A_{x_{n}}\right)+\int_{-\infty}^{+\infty} \frac{\beta(s) \Phi^{-1}\left(A_{x_{n}}+\int_{s}^{+\infty} f\left(r, x_{n}(r), x_{n}^{\prime}(r)\right) d r\right)}{\rho(s) a\left(s, x_{n}(s), x_{n}^{\prime}(s)\right)} d s \\
-\int_{-\infty}^{+\infty} h\left(r, x_{n}(r), x_{n}^{\prime}(t)\right) d r=0 .
\end{gathered}
$$

Corresponding to $x_{n}(n=0,1,2, \ldots)$. Since $x_{n} \rightarrow x_{0}$ as $n \rightarrow \infty$, there exists an $M_{0}>0$ such that $\left\|x_{n}\right\| \leq M_{0}(n=0,1,2, \ldots)$. The fact $f, g, h$ are Carathédory functions means there exists $\phi_{M_{0}} \in L^{1}(R)$ such that

$$
\begin{aligned}
& f\left(t, x_{n}(t), x_{n}^{\prime}(t)\right)=f\left(t, x_{n}(t), \frac{1}{\rho(t)} \rho(t) x_{n}^{\prime}(t)\right) \leq \phi_{M_{0}}(t), \quad t \in R, \\
& g\left(t, x_{n}(t), x_{n}^{\prime}(t)\right) \leq \phi_{M_{0}}(t), \quad h\left(t, x_{n}(t), x_{n}^{\prime}(t)\right) \leq \phi_{M_{0}}(t), \quad t \in R .
\end{aligned}
$$

Then

$$
\begin{gathered}
\int_{-\infty}^{+\infty} f\left(r, x_{n}(r), x_{n}^{\prime}(r)\right) d r \leq \int_{-\infty}^{+\infty} \phi_{M_{0}}(r) d r<\infty \\
\int_{-\infty}^{+\infty} g\left(r, x_{n}(r), x_{n}^{\prime}(r)\right) d r \leq \int_{-\infty}^{+\infty} \phi_{M_{0}}(r) d r<\infty \\
\int_{-\infty}^{+\infty} h\left(r, x_{n}(r), x_{n}^{\prime}(r)\right) d r \leq \int_{-\infty}^{+\infty} \phi_{M_{0}}(r) d r<\infty .
\end{gathered}
$$

So, by $(2.2)$, we have

$$
\begin{aligned}
\left|A_{x_{n}}\right| & \leq \int_{-\infty}^{+\infty}\left|f\left(r, x(r), x^{\prime}(r)\right)\right| d s+\Phi\left(\frac{\int_{-\infty}^{+\infty}\left|h\left(r, x(r), x^{\prime}(r)\right)\right| d r}{1+\int_{-\infty}^{+\infty} \frac{\beta(r)}{M \rho(r)} d r}\right) \\
& \leq \int_{-\infty}^{+\infty} \phi_{M_{0}}(s) d s+\Phi\left(\frac{\int_{-\infty}^{+\infty} \phi_{M_{0}}(s) d s}{1+\int_{-\infty}^{+\infty} \frac{\beta(r)}{M \rho(r)} d r}\right)
\end{aligned}
$$

which means that $\left\{A_{x_{n}}\right\}$ is uniformly bounded. It follows that

$$
\begin{aligned}
& \int_{-\infty}^{+\infty} \frac{\beta(s) \Phi^{-1}\left(A_{x_{n}}+\int_{s}^{+\infty} f\left(r, x_{n}(r), x_{n}^{\prime}(r)\right) d r\right)}{\rho(s) a\left(s, x_{n}(s), x_{n}^{\prime}(s)\right)} d s \\
& \quad \leq \frac{1}{m} \int_{-\infty}^{+\infty} \frac{\beta(s)}{\rho(s)} d s \Phi^{-1}\left(2 \int_{-\infty}^{+\infty} \phi_{M_{0}}(r) d r+\Phi\left(\frac{\int_{-\infty}^{+\infty} \phi_{M_{0}}(s) d s}{1+\int_{-\infty}^{+\infty} \frac{\beta(s)}{M \rho(s)} d s}\right)\right) .
\end{aligned}
$$


Suppose that $\left\{A_{x_{n}}\right\}$ does not converge to $A_{x_{0}}$. Then there exist two subsequences $\left\{A_{x_{n_{k}}(1)}\right\}$ and $\left\{A_{x_{n_{k}}(2)}\right\}$ of $\left\{A_{x_{n}}\right\}$ with $A_{x_{n_{k}}(1)} \rightarrow c_{1}$ and $A_{x_{n_{k}}(2)} \rightarrow c_{2}$ as $k \rightarrow \infty$, but $c_{1} \neq c_{2}$. By the construction of $A_{x_{n}}(n=1,2, \ldots)$, we have

$$
\begin{aligned}
& \Phi^{-1}\left(A_{x_{n_{k}}^{(1)}}\right)+\int_{-\infty}^{+\infty} \frac{\beta(s) \Phi^{-1}\left(A_{x_{n_{k}}^{(1)}}+\int_{s}^{+\infty} f\left(r, x_{n_{k}}^{(1)}(r), x_{n_{k}}^{(1)^{\prime}}(r)\right) d r\right)}{\rho(s) a\left(s, x_{n_{k}}^{(1)}(s), x_{n_{k}}^{(1)^{\prime}}(s)\right)} d s \\
& -\int_{-\infty}^{+\infty} h\left(r, x_{n_{k}}^{(1)}(r), x_{n_{k}}^{(1)^{\prime}}(t)\right) d r=0 .
\end{aligned}
$$

Let $k \rightarrow \infty$, using Lebesgue's dominated convergence theorem, the above equality implies

$$
\begin{gathered}
\Phi^{-1}\left(A_{x_{0}}\right)+\int_{-\infty}^{+\infty} \frac{\beta(s) \Phi^{-1}\left(A_{x_{0}}+\int_{s}^{+\infty} f\left(r, x_{0}(r), x_{0}^{\prime}(r)\right) d r\right)}{\rho(s) a\left(s, x_{0}(s), x_{0}^{\prime}(s)\right)} d s \\
-\int_{-\infty}^{+\infty} h\left(r, x_{0}(r), x_{0}^{\prime}(t)\right) d r=0 .
\end{gathered}
$$

Since $\left\{A_{x_{0}}\right\}$ is unique with respect to $x_{0}$, we get $c_{1}=A_{x_{0}}$. Similarly, $c_{2}=A_{x_{0}}$. Thus $c_{1}=c_{2}$, a contradiction. So, for any $x_{n} \rightarrow x_{0}$, one has $A_{x_{n}} \rightarrow A_{x_{0}}$, which means $A_{x}: X \rightarrow R$ is continuous.

Step 2. We show that $T$ is continuous on $X$. Since $A_{x}$ is continuous, then $B_{x}$ is continuous too. From the continuity of $A_{x}$ and $B_{x}$, and since $f, g, h$ are Caratheodory functions, the result follows.

To prove that $T$ maps bounded sets into relatively compact sets, we must prove that $T D$ is relative compact. Recall $W \subset X$ is relatively compact if

(i) it is bounded,

(ii) both $\left\{\frac{T x}{1+\tau(t)}: x \in W\right\}$ and $\left\{\rho(t)(T x)^{\prime}: x \in W\right\}$ are equi-continuous on any closed subinterval of $(-\infty,+\infty)$,

(iii) both $\left\{\frac{T x}{1+\tau(t)}: x \in W\right\}$ and $\left\{\rho(t)(T x)^{\prime}: x \in W\right\}$ are equi-convergent at $t=-\infty$,

(iv) both $\left\{\frac{T x}{1+\tau(t)}: x \in W\right\}$ and $\left\{\rho(t)(T x)^{\prime}: x \in W\right\}$ are equi-convergent at $t=+\infty$.

Hence we must do the following three steps.

Step 3. We show that $T$ maps bounded subsets into bounded sets. Let $D \subseteq X$ be a given bounded set. Then, there exists $M_{0}>0$ such that $D \subseteq\{x \in$ $\left.X:\|x\| \leq M_{0}\right\}$. Then there exists $\phi_{M_{0}} \in L^{1}(R)$ such that

$$
\begin{aligned}
& \left|f\left(t, x(t), x^{\prime}(t)\right)\right|=\left|f\left(t, x(t), \frac{1}{\rho(t)} \rho(t) x^{\prime}(t)\right)\right| \leq \phi_{M_{0}}(t), \quad t \in R, \\
& \left|g\left(t, x(t), x^{\prime}(t)\right)\right| \leq \phi_{M_{0}}(t), \quad\left|h\left(t, x(t), x^{\prime}(t)\right)\right| \leq \phi_{M_{0}}(t), \quad t \in R .
\end{aligned}
$$


So

$$
\begin{aligned}
& \int_{-\infty}^{+\infty}\left|f\left(r, x(r), x^{\prime}(r)\right)\right| d r \leq \int_{-\infty}^{+\infty} \phi_{M_{0}}(r) d r<\infty \\
& \int_{-\infty}^{+\infty}\left|g\left(r, x(r), x^{\prime}(r)\right)\right| d r \leq \int_{-\infty}^{+\infty} \phi_{M_{0}}(r) d r<\infty \\
& \int_{-\infty}^{+\infty}\left|h\left(r, x(r), x^{\prime}(r)\right)\right| d r \leq \int_{-\infty}^{+\infty} \phi_{M_{0}}(r) d r<\infty
\end{aligned}
$$

Similarly we have

$$
\begin{aligned}
\left|A_{x}\right| \leq & \int_{-\infty}^{+\infty} \phi_{M_{0}}(s) d s+\Phi\left(\frac{\int_{-\infty}^{+\infty} \phi_{M_{0}}(s) d s}{1+\int_{-\infty}^{+\infty} \frac{\beta(r)}{M \rho(r)} d r}\right)=: M_{1}<\infty \\
\left|B_{x}\right|= & \mid \frac{\Phi^{-1}\left(A_{x}+\int_{-\infty}^{+\infty} f\left(r, x(r), x^{\prime}(r)\right) d r\right)-\int_{-\infty}^{+\infty} g\left(r, x(r), x^{\prime}(r)\right) d r}{\int_{-\infty}^{+\infty} \alpha(s) d s} \\
& -\frac{\int_{0}^{+\infty} \alpha(s) \int_{0}^{s} \frac{\Phi^{-1}\left(A_{x}+\int_{u}^{+\infty} f\left(r, x(r), x^{\prime}(r)\right) d r\right)}{\rho(u) a\left(u, x(u), x^{\prime}(u)\right)} d u d s}{\int_{-\infty}^{+\infty} \alpha(s) d s} \\
& +\frac{\int_{-\infty}^{0} \alpha(s) \int_{s}^{0} \frac{\Phi^{-1}\left(A_{x}+\int_{u}^{+\infty} f\left(r, x(r), x^{\prime}(r)\right) d r\right)}{\rho(u) a\left(u, x(u), x^{\prime}(u)\right)} d u d s}{\int_{-\infty}^{+\infty} \alpha(s) d s} \mid \\
\leq & \frac{\Phi^{-1}\left(M_{1}+\int_{-\infty}^{+\infty} \phi_{M_{0}}(r) d r\right)+\int_{-\infty}^{+\infty} \phi_{M_{0}}(r) d r}{\int_{-\infty}^{+\infty} \alpha(s) d s} \\
& +\frac{\int_{0}^{+\infty} \alpha(s) \int_{0}^{s} \frac{1}{\rho(u)} d u d s \Phi^{-1}\left(M_{1}+\int_{-\infty}^{+\infty} \phi_{M_{0}}(r) d r\right)}{m \int_{-\infty}^{+\infty} \alpha(s) d s} \\
& +\frac{\int_{-\infty}^{0} \alpha(s)\left|\int_{0}^{s} \frac{1}{\rho(u)} d u\right| d s \Phi^{-1}\left(M_{1}+\int_{-\infty}^{+\infty} \phi_{M_{0}}(r) d r\right)}{m \int_{-\infty}^{+\infty} \alpha(s) d s}=: M_{2}<+\infty
\end{aligned}
$$

Therefore,

$$
\begin{aligned}
\frac{|(T x)(t)|}{1+\tau(t)} & =\left\{\begin{array}{l}
\frac{1}{1+\tau(t)}\left|B_{x}+\int_{0}^{t} \frac{\Phi^{-1}\left(A_{x}+\int_{s}^{+\infty} f\left(r, x(r), x^{\prime}(r)\right) d r\right)}{\rho(s) a\left(s, x(s), x^{\prime}(s)\right)} d s\right|, \quad t \geq 0, \\
\frac{1}{1+\tau(t)}\left|B_{x}-\int_{t}^{0} \frac{\Phi^{-1}\left(A_{x}+\int_{s}^{+\infty} f\left(r, x(r), x^{\prime}(r)\right) d r\right)}{\rho(s) a\left(s, x(s), x^{\prime}(s)\right)} d s\right|, \quad t \leq 0,
\end{array}\right. \\
& \leq\left\{\begin{array}{l}
M_{2}+\frac{1}{m} \frac{1}{1+\tau(t)} \int_{0}^{t} \frac{1}{\rho(s)} d s \Phi^{-1}\left(M_{1}+\int_{s}^{+\infty} \phi_{M_{0}}(r) d r\right), \quad t \geq 0, \\
M_{2}+\frac{1}{m} \frac{1}{1+\tau(t)} \int_{t}^{0} \frac{1}{\rho(s)} d s \Phi^{-1}\left(M_{1}+\int_{s}^{+\infty} \phi_{M_{0}}(r) d r\right), \quad t \leq 0,
\end{array}\right. \\
& \leq\left\{\begin{array}{l}
M_{2}+\frac{1}{m} \Phi^{-1}\left(M_{1}+\int_{-\infty}^{+\infty} \phi_{M_{0}}(r) d r\right), \quad t \geq 0, \\
M_{2}+\frac{1}{m} \Phi^{-1}\left(M_{1}+\int_{-\infty}^{+\infty} \phi_{M_{0}}(r) d r\right), \quad t \leq 0,
\end{array}\right. \\
& =: M_{3}<+\infty .
\end{aligned}
$$


On the other hand, we have

$$
\begin{aligned}
\rho(t)\left|(T x)^{\prime}(t)\right| & =\frac{\left|\Phi^{-1}\left(A_{x}+\int_{t}^{\infty} f\left(u, x(u), x^{\prime}(u)\right) d u\right)\right|}{a\left(t, x(t), x^{\prime}(t)\right)} \\
& \leq \frac{1}{m} \Phi^{-1}\left(M_{1}+\int_{-\infty}^{+\infty} \phi_{M_{0}}(r) d r\right)=: M_{4} .
\end{aligned}
$$

Then

$$
\|(T x)\|=\max \left\{\sup _{t \in R} \frac{|(T x)(t)|}{1+\tau(t)}, \sup _{t \in R} \rho(t)\left|(T x)^{\prime}(t)\right|\right\} \leq \max \left\{M_{3}, M_{4}\right\}<+\infty .
$$

So, $\{T x: x \in D\}$ is bounded.

Step 4. Let $D$ be a bounded subset of $X$. We prove that both $\left\{\frac{T x}{1+\tau(t)}: x \in\right.$ $D\}$ and $\left\{\rho(T x)^{\prime}: x \in D\right\}$ are equi-continuous on each finite subinterval $[-K, K]$ on $R$. Suppose that $D \subset\left\{x \in X:\|x\| \leq M_{0}\right\}$. For any $K>0, t_{1}, t_{2} \in[-K, K]$ with $t_{1} \leq t_{2}$ and $x \in X$, since $f, g, h$ are Caratheodory functions, then there exists $\phi_{M_{0}} \in L^{1}(R)$ such that (2.7) and (2.8) hold. One sees that (2.6) holds.

First, we consider $\left|\rho\left(t_{1}\right)(T x)^{\prime}\left(t_{1}\right)-\rho\left(t_{2}\right)(T x)^{\prime}\left(t_{2}\right)\right|$. One sees that

$$
\begin{aligned}
& \left|\rho\left(t_{1}\right)(T x)^{\prime}\left(t_{1}\right)-\rho\left(t_{2}\right)(T x)^{\prime}\left(t_{2}\right)\right| \\
& =\left|\frac{\Phi^{-1}\left(A_{x}+\int_{t_{1}}^{+\infty} f\left(r, x(r), x^{\prime}(r)\right) d r\right)}{a\left(t_{1}, x\left(t_{1}\right), x^{\prime}\left(t_{1}\right)\right)}-\frac{\Phi^{-1}\left(A_{x}+\int_{t_{2}}^{+\infty} f\left(r, x(r), x^{\prime}(r)\right) d r\right)}{a\left(t_{2}, x\left(t_{2}\right), x^{\prime}\left(t_{2}\right)\right)}\right| \\
& \leq \frac{\left|\Phi^{-1}\left(A_{x}+\int_{t_{1}}^{+\infty} f\left(r, x(r), x^{\prime}(r)\right) d r\right)-\Phi^{-1}\left(A_{x}+\int_{t_{2}}^{+\infty} f\left(r, x(r), x^{\prime}(r)\right) d r\right)\right|}{a\left(t_{1}, x\left(t_{1}\right), x^{\prime}\left(t_{1}\right)\right)} \\
& +\left|\Phi^{-1}\left(A_{x}+\int_{t_{2}}^{+\infty} f\left(r, x(r), x^{\prime}(r)\right) d r\right)\right|\left|\frac{1}{a\left(t_{1}, x\left(t_{1}\right), x^{\prime}\left(t_{1}\right)\right)}-\frac{1}{a\left(t_{2}, x\left(t_{2}\right), x^{\prime}\left(t_{2}\right)\right)}\right| \\
& \leq \frac{\left|\Phi^{-1}\left(A_{x}+\int_{t_{1}}^{+\infty} f\left(r, x(r), x^{\prime}(r)\right) d r\right)-\Phi^{-1}\left(A_{x}+\int_{t_{2}}^{+\infty} f\left(r, x(r), x^{\prime}(r)\right) d r\right)\right|}{m} \mid . \\
& +\Phi^{-1}\left(M_{1}+\int_{-\infty}^{+\infty} \phi_{M_{0}}(r) d r\right)\left|\frac{1}{a\left(t_{1}, x\left(t_{1}\right), x^{\prime}\left(t_{1}\right)\right)}-\frac{1}{a\left(t_{2}, x\left(t_{2}\right), x^{\prime}\left(t_{2}\right)\right)}\right|
\end{aligned}
$$

Since

$$
\left|A_{x}+\int_{t}^{+\infty} f\left(r, x(r), x^{\prime}(r)\right) d r\right| \leq \int_{-\infty}^{+\infty} \phi_{M_{0}}(r) d r+M_{1}=: r
$$

and $\Phi^{-1}(s)$ is uniformly continuous on $[-r, r]$, then for each $\epsilon>0$ there exists $\mu>0$ such that $\left|s_{1}-s_{2}\right|<\mu$ with $s_{1}, s_{2} \in[-r, r]$ implies that $\mid \Phi^{-1}\left(s_{1}\right)-$ $\Phi^{-1}\left(s_{2}\right) \mid<m / 2 \epsilon$. Since

$$
\begin{gathered}
\left|\Phi\left(\rho\left(t_{1}\right) a\left(t_{1}, x\left(t_{1}\right), x^{\prime}\left(t_{1}\right)\right)(T x)^{\prime}\left(t_{1}\right)\right)-\Phi\left(\rho\left(t_{2}\right) a\left(t_{2}, x\left(t_{2}\right), x^{\prime}\left(t_{2}\right)\right)(T x)^{\prime}\left(t_{2}\right)\right)\right| \\
=\left|\int_{t_{2}}^{t_{1}} f\left(r, x(r), x^{\prime}(r)\right) d r\right| \leq \int_{t_{1}}^{t_{2}} \phi_{M}(r) d r \rightarrow 0 \quad \text { uniformly as } t_{1} \rightarrow t_{2},
\end{gathered}
$$


then there exists $\sigma_{1}>0$ such that $\left|t_{2}-t_{1}\right|<\sigma_{1}$ implies that

$$
\begin{aligned}
& \mid \Phi\left(\rho\left(t_{1}\right) a\left(t_{1}, x\left(t_{1}\right), x^{\prime}\left(t_{1}\right)\right)(T x)^{\prime}\left(t_{1}\right)\right) \\
& \quad-\Phi\left(\rho\left(t_{2}\right) a\left(t_{2}, x\left(t_{2}\right), x^{\prime}\left(t_{2}\right)\right)(T x)^{\prime}\left(t_{2}\right)\right) \mid<\mu .
\end{aligned}
$$

Thus $\left|t_{1}-t_{2}\right|<\sigma_{1}$ implies that

$$
\begin{aligned}
\left|\rho\left(t_{1}\right) a\left(t_{1}, x\left(t_{1}\right), x^{\prime}\left(t_{1}\right)\right)(T x)^{\prime}\left(t_{1}\right)-\rho\left(t_{2}\right) a\left(t_{2}, x\left(t_{2}\right), x^{\prime}\left(t_{2}\right)\right)(T x)^{\prime}\left(t_{2}\right)\right| & =\mid \Phi^{-1}\left(\Phi\left(\rho\left(t_{1}\right) a\left(t_{1}, x\left(t_{1}\right), x^{\prime}\left(t_{1}\right)\right)(T x)^{\prime}\left(t_{1}\right)\right)\right) \\
& -\Phi^{-1}\left(\Phi\left(\rho\left(t_{2}\right) a\left(t_{2}, x\left(t_{2}\right), x^{\prime}\left(t_{2}\right)\right)(T x)^{\prime}\left(t_{2}\right)\right)\right) \mid \\
= & \mid \Phi^{-1}\left(A_{x}+\int_{t_{1}}^{+\infty} f\left(r, x(r), x^{\prime}(r)\right) d r\right) \\
& -\Phi^{-1}\left(A_{x}+\int_{t_{2}}^{+\infty} f\left(r, x(r), x^{\prime}(r)\right) d r\right) \mid<\frac{m}{2} \epsilon .
\end{aligned}
$$

Since $1 / a\left(t, x(t), x^{\prime}(t)\right)$ is uniformly continuous on $[-K, K]$, then there exists $\sigma_{2}>0$ such that $\left|t_{2}-t_{1}\right|<\sigma_{2}$ implies

$$
\left|\frac{1}{a\left(t_{1}, x\left(t_{1}\right), x^{\prime}\left(t_{1}\right)\right)}-\frac{1}{a\left(t_{2}, x\left(t_{2}\right), x^{\prime}\left(t_{2}\right)\right)}\right|<\frac{1}{\Phi^{-1}\left(M_{1}+\int_{-\infty}^{+\infty} \phi_{M_{0}}(r) d r\right)} \frac{\epsilon}{2} .
$$

Hence $\left|t_{1}-t_{2}\right|<\min \left\{\sigma_{1}, \sigma_{2}\right\}$ with $t_{1}, t_{2} \in[-K, K]$ implies that

$$
\left|\rho\left(t_{1}\right)(T x)^{\prime}\left(t_{1}\right)-\rho\left(t_{2}\right)(T x)^{\prime}\left(t_{2}\right)\right|<\epsilon .
$$

Now, we consider $\left|(T x)\left(t_{1}\right) /\left(1+\tau\left(t_{1}\right)\right)-(T x)\left(t_{2}\right) /\left(1+\tau\left(t_{2}\right)\right)\right|$.

Case 1. $0 \leq t_{1} \leq t_{2} \leq K$. By (2.3), we have

$$
\begin{aligned}
& \left|\frac{(T x)\left(t_{1}\right)}{1+\tau\left(t_{1}\right)}-\frac{(T x)\left(t_{2}\right)}{1+\tau\left(t_{2}\right)}\right| \leq\left|B_{x}\right|\left|\frac{1}{1+\tau\left(t_{1}\right)}-\frac{1}{1+\tau\left(t_{2}\right)}\right| \\
& +\left|\frac{\int_{0}^{t_{1}} \frac{\Phi^{-1}\left(A_{x}+\int_{s}^{+\infty} f\left(r, x(r), x^{\prime}(r)\right) d r\right)}{\rho(s) a\left(s, x(s), x^{\prime}(s)\right)} d s}{1+\tau\left(t_{1}\right)}-\frac{\int_{0}^{t_{2}} \frac{\Phi^{-1}\left(A_{x}+\int_{s}^{+\infty} f\left(r, x(r), x^{\prime}(r)\right) d r\right)}{\rho(s) a\left(s, x(s), x^{\prime}(s)\right)} d s}{1+\tau\left(t_{2}\right)}\right| \\
& \leq M_{2}\left|\tau\left(t_{1}\right)-\tau\left(t_{2}\right)\right|+\frac{1}{1+\tau\left(t_{1}\right)} \frac{1}{m} \int_{t_{1}}^{t_{2}} \frac{1}{\rho(s)} d s \Phi^{-1}\left(\int_{-\infty}^{+\infty} \phi_{M_{0}}(r) d r+M_{1}\right) \\
& \quad+\left|\frac{1}{1+\tau\left(t_{1}\right)}-\frac{1}{1+\tau\left(t_{2}\right)}\right| \int_{0}^{t_{2}} \frac{\Phi^{-1}\left(A_{x}+\int_{s}^{+\infty} f\left(r, x(r), x^{\prime}(r)\right) d r\right)}{\rho(s) a\left(s, x(s), x^{\prime}(s)\right)} d s \\
& \leq M_{2}\left|\tau\left(t_{1}\right)-\tau\left(t_{2}\right)\right|+\frac{1}{m} \int_{t_{1}}^{t_{2}} \frac{1}{\rho(s)} d s \Phi^{-1}\left(\int_{-\infty}^{+\infty} \phi_{M_{0}}(r) d r+M_{1}\right) \\
& \quad+\frac{1}{m}\left|\frac{1}{1+\tau\left(t_{1}\right)}-\frac{1}{1+\tau\left(t_{2}\right)}\right| \int_{0}^{t_{2}} \frac{1}{\rho(s)} d s \Phi^{-1}\left(\int_{-\infty}^{+\infty} \phi_{M_{0}}(r) d r+M_{1}\right) \\
& \leq M_{2}\left|\tau\left(t_{1}\right)-\tau\left(t_{2}\right)\right|+\frac{1}{m} \int_{t_{1}}^{t_{2}} \frac{1}{\rho(s)} d s \Phi^{-1}\left(\int_{-\infty}^{+\infty} \phi_{M_{0}}(r) d r+M_{1}\right) \\
& \quad+\frac{1}{m}\left|\tau\left(t_{1}\right)-\tau\left(t_{2}\right)\right| \Phi^{-1}\left(\int_{-\infty}^{+\infty} \phi_{M_{0}}(r) d r+M_{1}\right) .
\end{aligned}
$$


Case 2. $-K \leq t_{1} \leq t_{2} \leq 0$. We have similarly that

$$
\begin{aligned}
& \left|\frac{(T x)\left(t_{1}\right)}{1+\tau\left(t_{1}\right)}-\frac{(T x)\left(t_{2}\right)}{1+\tau\left(t_{2}\right)}\right| \\
& \quad \leq M_{2}\left|\tau\left(t_{1}\right)-\tau\left(t_{2}\right)\right|+\frac{1}{m} \int_{t_{1}}^{t_{2}} \frac{1}{\rho(s)} d s \Phi^{-1}\left(\int_{-\infty}^{+\infty} \phi_{M_{0}}(r) d r+M_{1}\right) \\
& \quad+\frac{1}{m}\left|\tau\left(t_{1}\right)-\tau\left(t_{2}\right)\right| \Phi^{-1}\left(\int_{-\infty}^{+\infty} \phi_{M_{0}}(r) d r+M_{1}\right) .
\end{aligned}
$$

Case 3. $-K \leq t_{1} \leq t_{2} \leq K$. We have

$$
\begin{aligned}
& \left|\frac{(T x)\left(t_{1}\right)}{1+\tau\left(t_{1}\right)}-\frac{(T x)\left(t_{2}\right)}{1+\tau\left(t_{2}\right)}\right| \\
& \quad=\left|B_{x}\right|\left|\frac{1}{1+\tau\left(t_{1}\right)}-\frac{1}{1+\tau\left(t_{2}\right)}\right| \\
& \quad+\left|\frac{\int_{t_{1}}^{0} \frac{\Phi^{-1}\left(A_{x}+\int_{s}^{+\infty} f\left(r, x(r), x^{\prime}(r)\right) d r\right)}{\rho(s) a\left(s, x(s), x^{\prime}(s)\right)} d s}{1+\tau\left(t_{1}\right)}+\frac{\int_{0}^{t_{2}} \frac{\Phi^{-1}\left(A_{x}+\int_{s}^{+\infty} f\left(r, x(r), x^{\prime}(r)\right) d r\right)}{\rho(s) a\left(s, x(s), x^{\prime}(s)\right)} d s}{1+\tau\left(t_{2}\right)}\right| \\
& \quad \leq M_{2}\left|\tau\left(t_{1}\right)-\tau\left(t_{2}\right)\right|+\frac{1}{m} \int_{t_{1}}^{t_{2}} \frac{1}{\rho(s)} d s \Phi^{-1}\left(\int_{-\infty}^{+\infty} \phi_{M_{0}}(r) d r+M_{1}\right) \\
& \quad+\frac{1}{m}\left|\tau\left(t_{1}\right)-\tau\left(t_{2}\right)\right| \Phi^{-1}\left(\int_{-\infty}^{+\infty} \phi_{M_{0}}(r) d r+M_{1}\right) .
\end{aligned}
$$

From Cases 1-3, we get

$$
\frac{(T x)\left(t_{1}\right)}{1+\tau\left(t_{1}\right)}-\frac{(T x)\left(t_{2}\right)}{1+\tau\left(t_{2}\right)} \rightarrow 0 \text { uniformly as } t_{1} \rightarrow t_{2} .
$$

Then there exists $\sigma_{3}>0$ such that $\left|t_{1}-t_{2}\right|<\sigma_{3}$ with $t_{1}, t_{2} \in[-K, K]$ implies

$$
\left|\frac{(T x)\left(t_{1}\right)}{1+\tau\left(t_{1}\right)}-\frac{(T x)\left(t_{2}\right)}{1+\tau\left(t_{2}\right)}\right|<\epsilon .
$$

Then (2.9) and (2.10) imply that both $\left\{\frac{T x}{1+\tau(t)}: x \in D\right\}$ and $\left\{\rho(T x)^{\prime}: x \in D\right\}$ are equi-continuous on $[-K, K]$. So both $\left\{\frac{T x}{1+\tau(t)}: x \in D\right\}$ and $\left\{\rho(T x)^{\prime}: x \in D\right\}$ are equi-continuous on each finite subinterval on $R$.

Step 5. Let $D$ be a bounded subset of $X$. We show that both $\left\{\frac{T x}{1+\tau(t)}: x \in\right.$ $D\}$ and $\left\{\rho(T x)^{\prime}: x \in D\right\}$ are equi-convergent at $+\infty$ and $-\infty$ respectively.

$$
\begin{aligned}
& \left|\frac{(T x)(t)}{1+\tau(t)}-\frac{\Phi^{-1}\left(A_{x}\right)}{a_{+}}\right| \\
& \quad \leq \frac{\left|B_{x}\right|}{1+\tau(t)}+\left|\frac{\int_{0}^{t} \frac{\Phi^{-1}\left(A_{x}+\int_{s}^{+\infty} f\left(r, x(r), x^{\prime}(r)\right) d r\right)}{\rho(s) a\left(s, x(s), x^{\prime}(s)\right)} d s}{1+\tau(t)}-\frac{\Phi^{-1}\left(A_{x}\right)}{a_{+}}\right| \\
& \quad \leq \frac{M_{2}}{1+\tau(t)}+\left|\frac{\int_{0}^{t} \frac{\Phi^{-1}\left(A_{x}+\int_{s}^{+\infty} f\left(r, x(r), x^{\prime}(r)\right) d r\right)}{\rho(s) a\left(s, x(s), x^{\prime}(s)\right)} d s-\int_{0}^{t} \frac{\Phi^{-1}\left(A_{x}\right)}{\rho(s) a_{+}} d s-\frac{\Phi^{-1}\left(A_{x}\right)}{a_{+}}}{1+\tau(t)}\right| \\
& \quad \leq \frac{M_{2}+\Phi^{-1}\left(M_{1}\right) / a_{+}}{1+\tau(t)}+\frac{\int_{0}^{t} \frac{1}{\rho(s)}\left|\frac{\Phi^{-1}\left(A_{x}+\int_{s}^{+\infty} f\left(r, x(r), x^{\prime}(r)\right) d r\right)}{a\left(s, x(s), x^{\prime}(s)\right)}-\frac{\Phi^{-1}\left(A_{x}\right)}{a_{+}}\right| d s}{1+\tau(t)} .
\end{aligned}
$$


It is easy to know that there exists $T_{1}>0$ such that $t>T_{1}$ implies

$$
0<\frac{M_{2}+\Phi^{-1}\left(M_{1}\right) / a_{+}}{1+\tau(t)}<\frac{\epsilon}{2}, \quad t>T_{1} .
$$

Similarly to Step 4, we can get that $\Phi^{-1}\left(A_{x}+\int_{t}^{+\infty} f\left(r, x(r), x^{\prime}(r)\right) d r\right) \rightarrow$ $\Phi^{-1}\left(A_{x}\right)$ uniformly as $t \rightarrow+\infty$. Together with that

$$
a\left(t, x(t), x^{\prime}(t)\right)=a\left(t,(1+\tau(t)) \frac{x(t)}{1+\tau(t)}, \frac{1}{\rho(t)} \rho(t) x^{\prime}(t)\right) \rightarrow a_{+}
$$

uniformly as $t \rightarrow+\infty$, we know that

$$
\frac{\Phi^{-1}\left(A_{x}+\int_{t}^{+\infty} f\left(r, x(r), x^{\prime}(r)\right) d r\right)}{a\left(t, x(t), x^{\prime}(t)\right)}-\frac{\Phi^{-1}\left(A_{x}\right)}{a_{+}} \rightarrow 0 \quad \text { uniformly as } t \rightarrow+\infty .
$$

Then there exists $T_{2}>0$ such that

$$
\left|\frac{\Phi^{-1}\left(A_{x}+\int_{t}^{+\infty} f\left(r, x(r), x^{\prime}(r)\right) d r\right)}{a\left(t, x(t), x^{\prime}(t)\right)}-\frac{\Phi^{-1}\left(A_{x}\right)}{a_{+}}\right|<\frac{\epsilon}{2}, \quad t>T_{2} .
$$

Then

$$
\left|\rho(t)(T x)^{\prime}(t)-\frac{\Phi^{-1}\left(A_{x}\right)}{a_{+}}\right|<\frac{\epsilon}{2}, \quad t>T_{2} .
$$

Furthermore, $t>\max \left\{T_{1}, T_{2}\right\}=: T_{3}$ implies that

$$
\begin{aligned}
& \mid \frac{(T x)(t)}{1+}+(t) \frac{\Phi^{-1}\left(A_{x}\right)}{a_{+}} \mid \\
&< \frac{\epsilon}{2}+\frac{\int_{0}^{t} \frac{1}{\rho(s)}\left|\frac{\Phi^{-1}\left(A_{x}+\int_{s}^{+\infty} f\left(r, x(r), x^{\prime}(r)\right) d r\right)}{a\left(s, x(s), x^{\prime}(s)\right)}-\frac{\Phi^{-1}\left(A_{x}\right)}{a_{+}}\right| d s}{1+\tau(t)} \\
&= \frac{\epsilon}{2}+\frac{\int_{0}^{T_{3}} \frac{1}{\rho(s)}\left|\frac{\Phi^{-1}\left(A_{x}+\int_{s}^{+\infty} f\left(r, x(r), x^{\prime}(r)\right) d r\right)}{a\left(s, x(s), x^{\prime}(s)\right)}-\frac{\Phi^{-1}\left(A_{x}\right)}{a_{+}}\right| d s}{1+\tau(t)} \\
& \quad+\frac{\int_{T_{3}}^{t} \frac{1}{\rho(s)}\left|\frac{\Phi^{-1}\left(A_{x}+\int_{s}^{+\infty} f\left(r, x(r), x^{\prime}(r)\right) d r\right)}{a\left(s, x(s), x^{\prime}(s)\right)}-\frac{\Phi^{-1}\left(A_{x}\right)}{a_{+}}\right| d s}{1+\tau(t)} \\
& \leq \frac{\epsilon}{2}+\frac{\int_{0}^{T_{3}} \frac{1}{\rho(s)} d s\left(\frac{\Phi^{-1}\left(M_{1}+\int_{\infty}^{+\infty} \phi_{M_{0}}(r) d r\right)}{m}+\frac{\Phi^{-1}\left(M_{1}\right)}{a_{+}}\right)}{1+\tau(t)}+\frac{\epsilon}{2} \frac{\int_{T_{3}}^{t} \frac{1}{\rho(s)} d s}{1+\tau(t)} .
\end{aligned}
$$

It is easy to see that there exists $T_{4}>T_{3}$ such that

$$
\frac{\int_{0}^{T_{3}} \frac{1}{\rho(s)} d s\left(\frac{\Phi^{-1}\left(M_{1}+\int_{-\infty}^{+\infty} \phi_{M_{0}}(r) d r\right)}{m}+\frac{\Phi^{-1}\left(M_{1}\right)}{a_{+}}\right)}{1+\tau(t)}<\epsilon, \quad t>T_{4} .
$$

Hence

$$
\left|\frac{(T x)(t)}{1+\tau(t)}-\frac{\Phi^{-1}\left(A_{x}\right)}{a_{+}}\right|<\frac{\epsilon}{2}+\epsilon+\frac{\epsilon}{2}=2 \epsilon, \quad t>T_{4} .
$$


So (2.11) and (2.12) imply that both $\left\{\rho(T x)^{\prime}: x \in D\right\}$ and $\left\{\frac{T x}{1+\tau(t)}: x \in D\right\}$ are equi-convergent at $+\infty$.

Similarly we can prove that both $\left\{\frac{T x}{1+\tau(t)}: x \in D\right\}$ and $\left\{\rho(T x)^{\prime}: x \in D\right\}$ are equi-convergent at $-\infty$. The details are omitted.

From Steps $3-5$, we see that $T$ maps bounded sets into relatively compact sets. Therefore, the operator $T: X \rightarrow X$ is completely continuous. The proof is complete.

\section{Main Theorems}

In this section, the main results on the existence of solutions of BVP (1.7) are established.

Let $L$ and $L_{n}$ be defined in Section 1. For nonnegative functions $a, b, c, a_{1}$, $b_{1}, c_{1}$ and $a_{2}, b_{2}, c_{2} \in L^{1}(R)$, we denote

$$
\begin{aligned}
\sigma_{0}= & \frac{1}{m}+\frac{1+\int_{0}^{+\infty} \alpha(s) \int_{0}^{s} \frac{1}{\rho(u)} d u d s+\int_{-\infty}^{0} \alpha(s) \int_{s}^{0} \frac{1}{\rho(u)} d u d s}{m \int_{-\infty}^{+\infty} \alpha(s) d s}, \\
\Delta_{1}= & \frac{\int_{-\infty}^{+\infty}\left[b_{1}(r)+c_{1}(r)\right] d r}{\int_{-\infty}^{+\infty} \alpha(s) d s}+\sigma_{0} L_{2} \frac{\int_{-\infty}^{+\infty}\left[b_{2}(r)+c_{2}(r)\right] d r}{1+\int_{-\infty}^{+\infty} \frac{\beta(s)}{M \rho(s)} d s} \\
& +\sigma_{0} L_{2} L_{3} L \Phi^{-1}\left(2 \int_{-\infty}^{+\infty} b(r) d r\right)+\sigma_{0} L_{2} L_{3} L \Phi^{-1}\left(2 \int_{-\infty}^{+\infty} c(r) d r\right), \\
\Delta_{2}= & L_{2} \frac{\int_{-\infty}^{+\infty}\left[b_{2}(r)+c_{2}(r)\right] d r}{m\left(1+\int_{-\infty}^{+\infty} \frac{\beta(s)}{M \rho(s)} d s\right)} \\
& +\left(L_{2} L_{3} L \Phi^{-1}\left(2 \int_{-\infty}^{+\infty} b(r) d r\right)+L_{2} L_{3} L \Phi^{-1}\left(2 \int_{-\infty}^{+\infty} c(r) d r\right)\right) / m .
\end{aligned}
$$

Theorem 1. Suppose that there exist nonnegative functions $a, b, c, a_{1}, b_{1}, c_{1}$ and $a_{2}, b_{2}, c_{2} \in L^{1}(R)$ satisfying $\Delta_{1}<1, \Delta_{2}<1$ and

$$
\begin{aligned}
& \left|f\left(t,(1+\tau(t)) x, \frac{1}{\rho(t)} y\right)\right| \leq a(t)+b(t) \Phi(|x|)+c(t) \Phi(|y|), \quad x, y \in R, t \in R, \\
& \left|g\left(t,(1+\tau(t)) x, \frac{1}{\rho(t)} y\right)\right| \leq a_{1}(t)+b_{1}(t)|x|+c_{1}(t)|y|, \quad x, y \in R, t \in R, \\
& \left|h\left(t,(1+\tau(t)) x, \frac{1}{\rho(t)} y\right)\right| \leq a_{2}(t)+b_{2}(t)|x|+c_{2}(t)|y|, \quad x, y \in R, t \in R .
\end{aligned}
$$

Then BVP (1.7) has at least one solution.

Proof. We will apply Lemma 1 to prove this theorem. Let $X$ and $T$ be defined in Section 2. From Lemma 3, $T: X \rightarrow X$ is a completely continuous operator. Let

$$
M_{5}=\max \left\{\frac{\int_{-\infty}^{+\infty} a_{1}(r) d r}{\int_{-\infty}^{+\infty} \alpha(s) d s}+\sigma_{0} L_{2} \frac{\int_{-\infty}^{+\infty} a_{2}(r) d r}{1+\int_{-\infty}^{+\infty} \frac{\beta(s)}{M \rho(s)} d s}++\sigma_{0} L_{2} L_{3}\right.
$$




$$
\left.\times \Phi^{-1}\left(2 \int_{-\infty}^{+\infty} a(r) d r\right), L_{2} \frac{\int_{-\infty}^{+\infty} a_{2}(r) d r}{m\left(1+\int_{-\infty}^{+\infty} \frac{\beta(s)}{M \rho(s)} d s\right)}+\frac{L_{2} L_{3} \Phi^{-1}\left(2 \int_{-\infty}^{+\infty} a(r) d r\right)}{m}\right\}
$$

Choose

$$
M_{0}>M_{5} /\left(1-\max \left\{\Delta_{1}, \Delta_{2}\right\}\right) .
$$

Now we define $\Omega=\left\{x \in X:\|x\|<M_{0}\right\}$. We will show that $T(\partial \Omega) \subset \bar{\Omega}$. In fact, if $x \in \partial \Omega$, with $\|T x\| \geq M_{0}$, then by definition of norm $\|\cdot\|$ we have

$$
0 \leq|x(t)| /(1+\tau(t)) \leq M_{0}, \quad \rho(t)\left|x^{\prime \prime}(t)\right| \leq M_{0}, \quad t \in R .
$$

By the definition of $T$, together with (2.4), we get

$$
\begin{aligned}
\left|B_{x}\right|= & \mid \frac{\Phi^{-1}\left(A_{x}+\int_{-\infty}^{+\infty} f\left(r, x(r), x^{\prime}(t)\right) d r\right)-\int_{-\infty}^{+\infty} g\left(r, x(r), x^{\prime}(r)\right) d r}{\int_{-\infty}^{+\infty} \alpha(s) d s} \\
& -\frac{\int_{0}^{+\infty} \alpha(s) \int_{0}^{s} \frac{\Phi^{-1}\left(A_{x}+\int_{u}^{+\infty} f\left(r, x(r), x^{\prime}(r)\right) d r\right)}{\rho(u) a\left(u, x(u), x^{\prime}(u)\right)} d u d s}{\int_{-\infty}^{+\infty} \alpha(s) d s} \\
& +\frac{\int_{-\infty}^{0} \alpha(s) \int_{s}^{0} \frac{\Phi^{-1}\left(A_{x}+\int_{u}^{+\infty} f\left(r, x(r), x^{\prime}(r)\right) d r\right)}{\rho(u) a\left(u, x(u), x^{\prime}(u)\right)} d u d s}{\int_{-\infty}^{+\infty} \alpha(s) d s} \mid \\
\leq & \frac{\int_{-\infty}^{+\infty}\left|g\left(r, x(r), x^{\prime}(r)\right)\right| d r}{\int_{-\infty}^{+\infty} \alpha(s) d s} \\
& +\frac{1+\int_{0}^{+\infty} \alpha(s) \int_{0}^{s} \frac{1}{\rho(u)} d u d s+\int_{-\infty}^{0} \alpha(s) \int_{s}^{0} \frac{1}{\rho(u)} d u d s}{m \int_{-\infty}^{+\infty} \alpha(s) d s} \\
& \times \Phi^{-1}\left(\Phi\left(\frac{\int_{-\infty}^{+\infty} \mid h\left(s, x(s), x^{\prime}(s)\right) d s}{1+\int_{-\infty}^{+\infty} \frac{\beta(s)}{M \rho(s)} d s}\right)+2 \int_{-\infty}^{+\infty} f\left(r, x(r), x^{\prime}(r)\right) d r\right) .
\end{aligned}
$$

Then

$$
\begin{aligned}
& \sup _{t \in R}|T x(t)| /(1+\tau(t)) \\
& =\sup _{t \in R} \begin{cases}\left|\frac{B_{x}}{1+\tau(t)}+\frac{\int_{0}^{t} \frac{\Phi^{-1}\left(A_{x}+\int_{s}^{+\infty} f\left(r, x(r), x^{\prime}(r)\right) d r\right)}{\rho(s) a\left(s, x(s), x^{\prime}(s)\right)} d s}{1+\tau(t)}\right|, \quad t \geq 0, \\
\left|\frac{B_{x}}{1+\tau(t)}-\frac{\int_{t}^{0} \frac{\Phi^{-1}\left(A_{x}+\int_{s}^{+\infty} f\left(r, x(r), x^{\prime}(r)\right) d r\right)}{\rho(s) a\left(s, x(s), x^{\prime}(s)\right)} d s}{1+\tau(t)}\right|, \quad t \leq 0,\end{cases} \\
& \leq \sup _{t \in R} \begin{cases}\frac{\left|B_{x}\right|}{1+\tau(t)}+\frac{\int_{0}^{t} \frac{\left|\Phi^{-1}\left(A_{x}+\int_{s}^{+\infty} f\left(r, x(r), x^{\prime}(r)\right) d r\right)\right|}{\rho(s) a\left(s, x(s), x^{\prime}(s)\right)} d s}{1+\tau(t)}, \quad t \geq 0, \\
\frac{\left|B_{x}\right|}{1+\tau(t)}+\frac{\int_{t}^{0} \frac{\left|\Phi^{-1}\left(A_{x}+\int_{s}^{+\infty} f\left(r, x(r), x^{\prime}(r)\right) d r\right)\right|}{\rho(s) a\left(s, x(s), x^{\prime}(s)\right)} d s}{1+\tau(t)}, \quad t \leq 0,\end{cases}
\end{aligned}
$$




$$
\begin{aligned}
& \leq \sup _{t \in R} \begin{cases}\left|B_{x}\right|+\frac{\int_{0}^{t} \frac{\left|\Phi^{-1}\left(A_{x}+\int_{s}^{+\infty} f\left(r, x(r), x^{\prime}(r)\right) d r\right)\right|}{\rho(s)} d s}{m(1+\tau(t))}, & t \geq 0, \\
\left|B_{x}\right|+\frac{\int_{t}^{0} \frac{\left|\Phi^{-1}\left(A_{x}+\int_{s}^{+\infty} f\left(r, x(r), x^{\prime}(r)\right) d r\right)\right|}{\rho(s)} d s}{m(1+\tau(t))}, \quad t \leq 0,\end{cases} \\
& \leq \sup _{t \in R} \begin{cases}\left|B_{x}\right|+\frac{\int_{0}^{t} \frac{1}{\rho(s)} d s\left|\Phi^{-1}\left(A_{x}+\int_{-\infty}^{+\infty} f\left(r, x(r), x^{\prime}(r)\right) d r\right)\right|}{m(1+\tau(t))}, & t \geq 0, \\
\left|B_{x}\right|+\frac{\int_{t}^{0} \frac{1}{\rho(s)} d s\left|\Phi^{-1}\left(A_{x}+\int_{-\infty}^{+\infty} f\left(r, x(r), x^{\prime}(r)\right) d r\right)\right|}{m(1+\tau(t))}, & t \leq 0,\end{cases} \\
& \leq\left|B_{x}\right|+\left|\Phi^{-1}\left(A_{x}+\int_{-\infty}^{+\infty} f\left(r, x(r), x^{\prime}(r)\right) d r\right)\right| / m \\
& \leq \int_{-\infty}^{+\infty}\left|g\left(r, x(r), x^{\prime}(r)\right)\right| d r / \int_{-\infty}^{+\infty} \alpha(s) d s \\
& +\left[\frac{1}{m}+\frac{1+\int_{0}^{+\infty} \alpha(s) \int_{0}^{s} \frac{1}{\rho(u)} d u d s+\int_{-\infty}^{0} \alpha(s)\left|\int_{0}^{s} \frac{1}{\rho(u)} d u\right| d s}{m \int_{-\infty}^{+\infty} \alpha(s) d s}\right] \\
& \times \Phi^{-1}\left(\Phi\left(\frac{\int_{-\infty}^{+\infty} \mid h\left(s, x(s), x^{\prime}(s)\right) d s}{1+\int_{-\infty}^{+\infty} \frac{\beta(s)}{M \rho(s)} d s}\right)+2 \int_{-\infty}^{+\infty} f\left(r, x(r), x^{\prime}(r)\right) d r\right) \\
& =\int_{-\infty}^{+\infty}\left|g\left(r, x(r), x^{\prime}(r)\right)\right| d r / \int_{-\infty}^{+\infty} \alpha(s) d s \\
& +\sigma_{0} \Phi^{-1}\left(\Phi\left(\frac{\int_{-\infty}^{+\infty} \mid h\left(s, x(s), x^{\prime}(s)\right) d s}{1+\int_{-\infty}^{+\infty} \frac{\beta(s)}{M \rho(s)} d s}\right)+2 \int_{-\infty}^{+\infty} f\left(r, x(r), x^{\prime}(r)\right) d r\right) \\
& \leq \int_{-\infty}^{+\infty}\left|g\left(r, x(r), x^{\prime}(r)\right)\right| d r / \int_{-\infty}^{+\infty} \alpha(s) d s \\
& +\sigma_{0} L_{2}\left[\frac{\int_{-\infty}^{+\infty} \mid h\left(s, x(s), x^{\prime}(s)\right) d s}{1+\int_{-\infty}^{+\infty} \frac{\beta(s)}{M \rho(s)} d s}+\Phi^{-1}\left(2 \int_{-\infty}^{+\infty} f\left(r, x(r), x^{\prime}(r)\right) d r\right)\right] \\
& \leq \int_{-\infty}^{+\infty}\left[a_{1}(r)+b_{1}(r) \frac{|x(r)|}{1+\tau(r)}+c_{1}(r) \rho(r)\left|x^{\prime}(r)\right|\right] d r / \int_{-\infty}^{+\infty} \alpha(s) d s \\
& +\sigma_{0} L_{2} \frac{\int_{-\infty}^{+\infty}\left[a_{2}(r)+b_{2}(r) \frac{|x(r)|}{1+\tau(r)}+c_{2}(r) \rho(r)\left|x^{\prime}(r)\right|\right] d r}{1+\int_{-\infty}^{+\infty} \frac{\beta(s)}{M \rho(s)} d s} \\
& +\sigma_{0} L_{2} \Phi^{-1}\left(2 \int_{-\infty}^{+\infty}\left[a(r)+b(r) \Phi\left(\frac{|x(r)|}{1+\tau(r)}\right)+c(r) \Phi\left(\rho(r)\left|x^{\prime}(r)\right|\right)\right] d r\right) \\
& \leq \int_{-\infty}^{+\infty}\left[a_{1}(r)+b_{1}(r)\|x\|+c_{1}(r)\|x\|\right] d r / \int_{-\infty}^{+\infty} \alpha(s) d s \\
& +\sigma_{0} L_{2} \int_{-\infty}^{+\infty}\left[a_{2}(r)+b_{2}(r)\|x\|+c_{2}(r)\|x\|\right] d r /\left(1+\int_{-\infty}^{+\infty} \frac{\beta(s)}{M \rho(s)} d s\right)
\end{aligned}
$$




$$
\begin{aligned}
& +\sigma_{0} L_{2} \Phi^{-1}\left(2 \int_{-\infty}^{+\infty}[a(r)+b(r) \Phi(\|x\|)+c(r) \Phi(\|x\|)] d r\right) \\
\leq & \int_{-\infty}^{+\infty}\left[a_{1}(r)+b_{1}(r)\|x\|+c_{1}(r)\|x\|\right] d r / \int_{-\infty}^{+\infty} \alpha(s) d s \\
& +\sigma_{0} L_{2} \int_{-\infty}^{+\infty}\left[a_{2}(r)+b_{2}(r)\|x\|+c_{2}(r)\|x\|\right] d r /\left(1+\int_{-\infty}^{+\infty} \frac{\beta(s)}{M \rho(s)} d s\right) \\
& +\sigma_{0} L_{2} L_{3} \Phi^{-1}\left(2 \int_{-\infty}^{+\infty} a(r) d r\right)+\sigma_{0} L_{2} L_{3} L \Phi^{-1}\left(2 \int_{-\infty}^{+\infty} b(r) d r\right)\|x\| \\
& +\sigma_{0} L_{2} L_{3} L \Phi^{-1}\left(2 \int_{-\infty}^{+\infty} c(r) d r\right)\|x\| \\
= & \frac{\int_{-\infty}^{+\infty} a_{1}(r) d r}{\int_{-\infty}^{+\infty} \alpha(s) d s}+\sigma_{0} L_{2} \frac{\int_{-\infty}^{+\infty} a_{2}(r) d r}{1+\int_{-\infty}^{+\infty} \frac{\beta(s)}{M \rho(s)} d s}+\sigma_{0} L_{2} L_{3} \Phi^{-1}\left(2 \int_{-\infty}^{+\infty} a(r) d r\right) \\
& +\left[\frac{\int_{-\infty}^{+\infty}\left[b_{1}(r)+c_{1}(r)\right] d r}{\int_{-\infty}^{+\infty} \alpha(s) d s}+\sigma_{0} L_{2} \frac{\int_{-\infty}^{+\infty}\left[b_{2}(r)+c_{2}(r)\right] d r}{1+\int_{-\infty}^{+\infty} \frac{\beta(s)}{M \rho(s)} d s}\right. \\
& \left.+\sigma_{0} L_{2} L_{3} L \Phi^{-1}\left(2 \int_{-\infty}^{+\infty} b(r) d r\right)+\sigma_{0} L_{2} L_{3} L \Phi^{-1}\left(2 \int_{-\infty}^{+\infty} c(r) d r\right)\right]\|x\| .
\end{aligned}
$$

It follows that

$$
\begin{aligned}
& \sup _{t \in R} \frac{|T x(t)|}{1+\tau(t)} \leq \frac{\int_{-\infty}^{+\infty} a_{1}(r) d r}{\int_{-\infty}^{+\infty} \alpha(s) d s}+\sigma_{0} L_{2} \frac{\int_{-\infty}^{+\infty} a_{2}(r) d r}{1+\int_{-\infty}^{+\infty} \frac{\beta(s)}{M \rho(s)} d s} \\
& \quad+\sigma_{0} L_{2} L_{3} \Phi^{-1}\left(2 \int_{-\infty}^{+\infty} a(r) d r\right)+\Delta_{1} M_{0}
\end{aligned}
$$

Similarly, we have

$$
\begin{aligned}
& \sup _{t \in R} \rho(t)\left|(T x)^{\prime}(t)\right| \leq \sup _{t \in R} \frac{\left|\Phi^{-1}\left(A_{x}+\int_{t}^{+\infty} f\left(r, x(r), x^{\prime}(r)\right) d r\right)\right|}{a\left(t, x(t), x^{\prime}(t)\right)} \\
& \leq\left(L_{2} \int_{-\infty}^{+\infty} a_{2}(r) d r /\left(1+\int_{-\infty}^{+\infty} \frac{\beta(s)}{M \rho(s)} d s\right)+L_{2} L L_{3} L \Phi^{-1}\left(2 \int_{-\infty}^{+\infty} a(r) d r\right)\right) / m \\
& \quad+\frac{\|x\|}{m}\left[L_{2} \int_{-\infty}^{+\infty}\left[b_{2}(r)+c_{2}(r)\right] d r /\left(1+\int_{-\infty}^{+\infty} \frac{\beta(s)}{M \rho(s)} d s\right)\right. \\
& \left.\quad+L_{2} L_{3} L \Phi^{-1}\left(2 \int_{-\infty}^{+\infty} b(r) d r\right)+L_{2} L_{3} L \Phi^{-1}\left(2 \int_{-\infty}^{+\infty} c(r) d r\right)\right]
\end{aligned}
$$

It follows that

$$
\begin{aligned}
\sup _{t \in R} \rho(t)\left|(T x)^{\prime}(t)\right| \leq & L_{2} \frac{\int_{-\infty}^{+\infty} a_{2}(r) d r}{m\left(1+\int_{-\infty}^{+\infty} \frac{\beta(s)}{M \rho(s)} d s\right)} \\
& +\frac{L_{2} L_{3} \Phi^{-1}\left(2 \int_{-\infty}^{+\infty} a(r) d r\right)}{m}+\Delta_{2} M_{0}
\end{aligned}
$$


Then (3.2) and (3.3) imply that

$$
\|T x\| \leq M_{5}+\max \left\{\Delta_{1}, \Delta_{2}\right\} M_{0}
$$

It follows from $\|T x\| \geq M_{0}$ that

$$
M_{0} \leq M_{5} /\left(1-\max \left\{\Delta_{1}, \Delta_{2}\right\}\right)
$$

a contradiction to (3.1). So $T(\partial \Omega) \subset \bar{\Omega}$. Thus Lemma 1 implies that the operator $T$ has at least one fixed point in $\Omega$. So BVP (1.7) has at least one solution.

Corollary 1. Suppose that there exists $r>0$ such that

$$
\begin{aligned}
& \int_{-\infty}^{+\infty}\left|g\left(t,(1+\tau(t)) x, \frac{1}{\rho(t)} y\right)\right| d t \leq \frac{r}{3} \int_{-\infty}^{+\infty} \alpha(s) d s \\
& \int_{-\infty}^{+\infty}\left|h\left(t,(1+\tau(t)) x, \frac{1}{\rho(t)} y\right)\right| d t \leq \frac{r}{3 \sigma_{0} L_{2}}\left(1+\int_{-\infty}^{+\infty} \frac{\beta(s)}{M \rho(s)} d s\right) \\
& \int_{-\infty}^{+\infty}\left|f\left(t,(1+\tau(t)) x, \frac{1}{\rho(t)} y\right)\right| d t \leq \frac{1}{2} \Phi\left(\frac{r}{3 \sigma_{0} L_{2}}\right),
\end{aligned}
$$

where $x, y \in[-r, r]$. Then BVP (1.7) has at least one solution.

Proof. From Lemma $3, T: X \rightarrow X$ is a completely continuous operator. Now we define $\Omega=\{x \in X:\|x\|<r\}$. For any $x \in \partial \Omega,\|x\|=r$. So

$$
\sup _{t \in R} \frac{|x(t)|}{1+\tau(t)} \leq r, \quad \sup _{t \in R} \rho(t)\left|x^{\prime}(t)\right| \leq r .
$$

By the assumptions, similarly to the proof of Theorem 1, we get

$$
\begin{aligned}
\sup _{t \in R} & \frac{|T x(t)|}{1+\tau(t)} \leq \frac{\int_{-\infty}^{+\infty}\left|g\left(r, x(r), x^{\prime}(r)\right)\right| d r}{\int_{-\infty}^{+\infty} \alpha(s) d s} \\
& \quad+\sigma_{0} L_{2}\left[\frac{\int_{-\infty}^{+\infty} \mid h\left(s, x(s), x^{\prime}(s)\right) d s}{1+\int_{-\infty}^{+\infty} \frac{\beta(s)}{M \rho(s)} d s}+\Phi^{-1}\left(2 \int_{-\infty}^{+\infty} f\left(r, x(r), x^{\prime}(r)\right) d r\right)\right] \\
\leq & \frac{r}{3}+\frac{r}{3}+\frac{r}{3}=r=\|x\| .
\end{aligned}
$$

Furthermore,

$$
\begin{aligned}
& \sup _{t \in R} \rho(t)\left|(T x)^{\prime}(t)\right| \\
& \quad \leq \frac{1}{m}\left(L_{2} \frac{\int_{-\infty}^{+\infty}\left|h\left(s, x(s), x^{\prime}(s)\right)\right| d s}{1+\int_{-\infty}^{+\infty} \frac{\beta(s)}{M \rho(s)} d s}+L_{2} \Phi^{-1}\left(2 \int_{-\infty}^{+\infty}\left|f\left(r, x(r), x^{\prime}(r)\right)\right| d r\right)\right)
\end{aligned}
$$




$$
\leq \frac{1}{m 3 \sigma_{0} L_{2}}\left(L_{2} \frac{r\left(1+\int_{-\infty}^{+\infty} \frac{\beta(s)}{M \rho(s)} d s\right)}{1+\int_{-\infty}^{+\infty} \frac{\beta(s)}{M \rho(s)} d s}+L_{2} r\right)=\frac{2 r}{3 m \sigma_{0}}<r=\|x\| .
$$

So $\|T x\| \leq\|x\|$ for all $x \in \partial \Omega$. Similar to the process in Theorem 1 , the result follows. The proof is complete.

Corollary 2. Suppose that

$$
\begin{aligned}
& \lim _{d \rightarrow+\infty} \frac{\max _{x, y \in[-d, d]} \int_{-\infty}^{+\infty}\left|f\left(s,(1+\tau(s)) x, \frac{1}{\rho(s)} y\right)\right| d s}{\Phi(d)}=0, \\
& \lim _{d \rightarrow+\infty} \frac{\max _{x, y \in[-d, d]} \int_{-\infty}^{+\infty}\left|g\left(s,(1+\tau(s)) x, \frac{1}{\rho(s)} y\right)\right| d s}{d}=0, \\
& \lim _{d \rightarrow+\infty} \frac{\max _{x, y \in[-d, d]} \int_{-\infty}^{+\infty}\left|h\left(s,(1+\tau(s)) x, \frac{1}{\rho(s)} y\right)\right| d s}{d}=0 .
\end{aligned}
$$

Then BVP (1.7) has at least one solution.

Proof. Let

$$
\varepsilon=\min \left\{\frac{1}{3} \int_{-\infty}^{+\infty} \alpha(s) d s, \frac{1}{3 \sigma_{0} L_{2}}\left(1+\int_{-\infty}^{+\infty} \frac{\beta(s)}{M \rho(s)} d s\right), \frac{1}{2} \Phi\left(\frac{1}{3 \sigma_{0} L_{2}}\right)\right\} .
$$

Then, there exists $r>0$, such that

$$
\begin{aligned}
& \int_{-\infty}^{+\infty}\left|g\left(s,(1+\tau(s)) x, \frac{1}{\rho(s)} y\right)\right| d s \leq \frac{r}{3} \int_{-\infty}^{+\infty} \alpha(s) d s \\
& \int_{-\infty}^{+\infty}\left|h\left(s,(1+\tau(s)) x, \frac{1}{\rho(s)} y\right)\right| d s \leq \frac{r}{3 \sigma_{0} L_{2}}\left(1+\int_{-\infty}^{+\infty} \frac{\beta(s)}{M \rho(s)} d s\right), \\
& \int_{-\infty}^{+\infty}\left|f\left(s,(1+\tau(s)) x, \frac{1}{\rho(s)} y\right)\right| d s \leq \frac{1}{2} \Phi\left(\frac{r}{3 \sigma_{0} L_{2}}\right) .
\end{aligned}
$$

By Corollary 1, BVP (1.7) has at least one solution. The proof is complete.

\section{An Example}

Now, we present an example to illustrate Theorem 1.

Example 1. Consider the following problem

$$
\begin{aligned}
& {\left[\Phi\left(e^{-|t|} a\left(t, x(t), x^{\prime}(t)\right) x^{\prime}(t)\right)\right]^{\prime}+\lambda\left[e^{-t^{2}}+\frac{1}{1+t^{2}}\left(1+\left|\int_{0}^{t} e^{|s|} d s\right|\right)^{-3}[x(t)]^{3}\right.} \\
& \left.\quad+\frac{|t|}{1+t^{4}} e^{-3|t|}\left[x^{\prime}(t)\right]^{3}\right]=0, t \in R \\
& \lim _{t \rightarrow-\infty} e^{t} a\left(t, x(t), x^{\prime}(t)\right) x^{\prime}(t)-\int_{-\infty}^{+\infty} e^{-2|s|} x(s) d s=0 \\
& \lim _{t \rightarrow+\infty} e^{-t} a\left(t, x(t), x^{\prime}(t)\right) x^{\prime}(t)+\int_{-\infty}^{+\infty} e^{-2|s|} x^{\prime}(s) d s=0
\end{aligned}
$$


where $\lambda \in R$ is a constant, $\Phi(x)=|x|^{2} x$ is a one-dimensional $p$-Lapalcian. Then BVP (4.1) has at least one solution if

$$
|\lambda|<\frac{8}{27 \pi(\sqrt[3]{2}+1)^{3}} .
$$

Proof. Corresponding to BVP (1.7), we have $\Phi(x)=|x|^{2} x, \rho(t)=e^{-|t|}$, with

$$
\begin{aligned}
& \tau(t)=\left|\int_{0}^{t} \frac{d s}{\rho(s)}\right|= \begin{cases}e^{t}-1, & t \geq 0, \\
-1+e^{-t}, & t \leq 0,\end{cases} \\
& a(t, x, y)=2+\frac{x^{2}}{(1+\tau(t))^{6}+x^{2}}+\frac{y^{2}}{e^{6|t|}+y^{2}}, \quad \alpha(t)=\beta(t)=e^{-2|t|}, \\
& f(t, x, y)=\lambda\left[e^{-t^{2}}+\frac{1}{1+t^{2}}\left(1+\left|\int_{0}^{t} e^{|s|} d s\right|\right)^{-3} x^{3}+\frac{|t|}{1+t^{4}} e^{-3|t|} y^{3}\right],
\end{aligned}
$$

and $g(t, x, y)=h(t, x, y) \equiv 0$.

One can show that

- $\rho \in C^{0}(R,[0,+\infty))$ with $\rho(t)>0$ for all $t \in R$ satisfies

$$
\int_{-\infty}^{0} \frac{1}{\rho(s)} d s=+\infty, \quad \int_{0}^{+\infty} \frac{1}{\rho(s)} d s=+\infty .
$$

We find that

- $a: R \times R \times R \rightarrow(0,+\infty)$ is continuous and satisfies

$$
2 \leq a(t,(1+\tau(t)) x, y / \rho(t)) \leq 4, \quad t \in R, x \in R, y \in R
$$

and for each $r>0,|x|,|y| \leq r$ imply that

$$
a(t,(1+\tau(t)) x, y / \rho(t))=2+\frac{x^{2}}{(1+\tau(t))^{4}+x^{2}}+\frac{y^{2}}{e^{4|t|}+y^{2}} \rightarrow a_{ \pm \infty}=2
$$

uniformly as $t \rightarrow \pm \infty$.

- $\alpha, \beta: R \rightarrow[0,+\infty)$ are continuous functions satisfying

$$
\begin{aligned}
& \int_{-\infty}^{+\infty} \alpha(s) d s>0, \quad \int_{0}^{+\infty} \alpha(s) \int_{0}^{s} \frac{d r}{\rho(r)} d s<+\infty \\
& \int_{-\infty}^{0} \alpha(s) \int_{s}^{0} \frac{d r}{\rho(r)} d s<+\infty, \quad \int_{-\infty}^{+\infty} \frac{\beta(s)}{\rho(s)} d s<+\infty .
\end{aligned}
$$

It is well known that $(s+t)^{\frac{1}{3}} \leq s^{\frac{1}{3}}+t^{\frac{1}{3}}$ for all $s, t \geq 0$.

- $\Phi(x)=|x|^{2} x$, is continuous and strictly increasing on $R, \Phi(0)=0$ and its inverse function is $\Phi^{-1}(x)=|x|^{-\frac{2}{3}} x$ for $x \neq 0$ and $\Phi^{-1}(0)=0$ is continuous too, moreover $\Phi^{-1}$ satisfies that there exist constants $L>0$ and $L_{n}>0$ such that $\Phi^{-1}\left(x_{1} x_{2}\right) \leq L \Phi^{-1}\left(x_{1}\right) \Phi^{-1}\left(x_{2}\right)$ with $L=1$ and

$$
\Phi^{-1}\left(x_{1}+\cdots+x_{n}\right) \leq L_{n}\left[\Phi^{-1}\left(x_{1}\right)+\cdots+\Phi^{-1}\left(x_{n}\right)\right]
$$

holds for all $x_{i} \geq 0(i=1,2, \ldots, n)$ with $L_{n}=1$. 
- $f, g, h$ defined on $R^{3}$ are nonnegative Caratheodory functions. To apply Theorem 1 , choose

$$
\begin{aligned}
& a(t)=|\lambda| e^{-t^{2}}, \quad b(t)=|\lambda| \frac{1}{1+t^{2}}, \quad c(t)=|\lambda| \frac{|t|}{1+t^{4}}, \\
& a_{1}(t)=a_{2}(t)=b_{1}(t)=b_{2}(t)=c_{1}(t)=c_{2}(t)=0 .
\end{aligned}
$$

It is easy to show that $a, b, c, a_{1}, b_{1}, c_{1}, a_{2}, b_{2}, c_{2} \in L^{1}(R)$ and

$$
\begin{aligned}
& \left|f\left(t,(1+\tau(t)) x, \frac{1}{\rho(t)} y\right)\right| \leq a(t)+b(t) \Phi(|x|)+c(t) \Phi(|y|), \quad x, y \in R, t \in R, \\
& \left|g\left(t,(1+\tau(t)) x, \frac{1}{\rho(t)} y\right)\right| \leq a_{1}(t)+b_{1}(t)|x|+c_{1}(t)|y|, \quad x, y \in R, t \in R, \\
& \left|h\left(t,(1+\tau(t)) x, \frac{1}{\rho(t)} y\right)\right| \leq a_{2}(t)+b_{2}(t)|x|+c_{2}(t)|y|, \quad x, y \in R, t \in R .
\end{aligned}
$$

By direct computation, we get

$$
\begin{aligned}
\sigma_{0}= & \frac{1}{m}+\frac{1+\int_{0}^{+\infty} \alpha(s) \int_{0}^{s} \frac{1}{\rho(u)} d u d s+\int_{-\infty}^{0} \alpha(s) \int_{s}^{0} \frac{1}{\rho(u)} d u d s}{m \int_{-\infty}^{+\infty} \alpha(s) d s}=\frac{3}{2} \\
\Delta_{1}= & \frac{\int_{-\infty}^{+\infty}\left[b_{1}(r)+c_{1}(r)\right] d r}{\int_{-\infty}^{+\infty} \alpha(s) d s}+\sigma_{0} L_{2} \frac{\int_{-\infty}^{+\infty}\left[b_{2}(r)+c_{2}(r)\right] d r}{1+\int_{-\infty}^{+\infty} \frac{\beta(s)}{M \rho(s)} d s} \\
& +\sigma_{0} L_{2} L_{3} L \Phi^{-1}\left(2 \int_{-\infty}^{+\infty} b(r) d r\right)+\sigma_{0} L_{2} L_{3} L \Phi^{-1}\left(2 \int_{-\infty}^{+\infty} c(r) d r\right) \\
= & \frac{3}{2}|\lambda|^{\frac{1}{3}}\left([2 \pi]^{\frac{1}{3}}+\pi^{\frac{1}{3}}\right), \\
\Delta_{2}= & L_{2} \frac{\int_{-\infty}^{+\infty}\left[b_{2}(r)+c_{2}(r)\right] d r}{m\left(1+\int_{-\infty}^{+\infty} \frac{\beta(s)}{M \rho(s)} d s\right)} \\
& +\frac{L_{2} L_{3} L \Phi^{-1}\left(2 \int_{-\infty}^{+\infty} b(r) d r\right)+L_{2} L_{3} L \Phi^{-1}\left(2 \int_{-\infty}^{+\infty} c(r) d r\right)}{m} \\
= & \frac{1}{2}|\lambda|^{\frac{1}{3}}\left([2 \pi]^{\frac{1}{3}}+\pi^{\frac{1}{3}}\right) .
\end{aligned}
$$

It follows from Theorem 1 that BVP (4.1) has at least one solution if

$$
|\lambda|<\frac{8}{27 \pi(\sqrt[3]{2}+1)^{3}} .
$$

\section{Acknowledgments}

The author thanks Alessandro Calamai for his great help in improving the exposition of the paper. The author also thanks the anonymous referees for their careful reading of this manuscript and for suggesting some useful stylistic changes. 


\section{References}

[1] R.P. Agarwal. Boundary Value Problems for Higher Order Differential Equations. World Scientific, Singapore, 1986.

[2] C. Avramescu and C. Vladimirescu. Limits of solutions of a perturbed linear differential equation. Electron. J. Qual. Theory Differ. Equ., 3:1-11, 2002.

[3] C. Avramescu and C. Vladimirescu. Existence of solutions to second order ordinary differential equations having finite limits at $\pm \infty$. Electron. J. Differential Equations, 18:1-12, 2004.

[4] C. Avramescu and C. Vladimirescu. Existence of homoclinic solutions to a nonlinear second order ODE, dynamics of continuous, discrete and impulsive systems. Ser. A, Math. Anal., 15:481-491, 2008.

[5] B. Bianconi and F. Papalini. Non-autonomous boundary value problems on the real line. Discrete Contin. Dyn. Syst., 15:759-776, 2006.

http://dx.doi.org/10.3934/dcds.2006.15.759.

[6] A. Cabada and J.A. Cid. Heteroclinic solutions for non-autonomous boundary value problems with singular $\Phi$-Laplacian operators. In Dynamical Systems, Differential Equations and Applications. 7th AIMS Conference, Discrete Contin. Dyn. Syst., suppl., pp. 118-122, 2009.

[7] A. Calamai. Heteroclinic solutions of boundary value problems on the real line involving singular $\Phi$-Laplacian operators. J. Math. Anal. Appl., 378:667-679, 2011. http://dx.doi.org/10.1016/j.jmaa.2011.01.056.

[8] J. Chamberlain, L. Kong and Q. Kong. Nodal solutions of nonlocal boundary value problems. Mathematical Modelling and Applications, 14:435-450, 2009.

[9] R. Čiegis, A. Štikonas, O. Štikonienè and O. Suboč. Stationary problems with nonlocal boundary conditions. Math. Model. Anal., 6(2):178-191, 2001.

[10] R. Čiegis and N. Tumanova. Numerical solution of parabolic problems with nonlocal boundary conditions. Numerical Functional Analysis and Optimization, 31(10):1318-1329, 2010.

[11] G. Cupini, C. Marcelli and F. Papalini. Heteroclinic solutions of boundary value problems on the real line involving general nonlinear differential operators. Differential Integral Equations, 24:619-644, 2011.

[12] G. Cupini, C. Marcelli and F. Papalini. On the solvability of a boundary value problem on the real line. Bound. Value Probl., 2011(26):1-17, 2011.

[13] K. Deimling. Nonlinear Functional Analysis. Springer, Berlin, Germany, 1985.

[14] W. Ge. Boundary Value Problems for Ordinary Differential Equations. Science Press, Beijing, 2007.

[15] V.A. Il'in and E.I. Moiseev. Nonlocal boundary-value problem of the second kind for a Sturm-Liouville operator. Differ. Equ., 23:979-987, 1987.

[16] C. Marcelli and F. Papalini. Heteroclinic connections for fully non-linear non-autonomous second-order differential equations. J. Differential Equations, 241:160-183, 2007. http://dx.doi.org/10.1016/j.jde.2007.05.038.

[17] C.G. Philos and I.K. Purnaras. A boundary value problem on the whole line to second order nonlinear differential equations. Georgian Math. J., 17:241-252, 2010 . 
[18] I. Rachunkova, S. Stanek and M. Tvrdy. Singularities and Laplacians in boundary value problems for nonlinear ordinary differential equations. In A. Canada, P. Drabek and A. Fonde(Eds.), Handbook of Differential Equations, Ordinary Differential Equations, vol. 3, pp. 606-723. Elsevier, 2006.

[19] Y. Wang and W. Ge. Existence of triple positive solutions for multi-point boundary value problems with a one dimensional p-Laplacian. Computers and Mathematics with Applications, 54:793-807, 2007. 